\title{
Extragalactic globular clusters in the near-infrared
}

\section{The globular clusters systems of NGC 3115 and NGC $4365^{\star, \star \star}$}

\author{
T. H. Puzia ${ }^{1}$, S. E. Zepf ${ }^{2,3}$, M. Kissler-Patig ${ }^{4}$, M. Hilker ${ }^{5}$, D. Minniti ${ }^{6}$, and P. Goudfrooij ${ }^{7}$ \\ 1 Sternwarte der Ludwig-Maximilians-Universität, Scheinerstr. 1, 81679 München, Germany \\ e-mail: puzia@usm.uni-muenchen.de \\ 2 Department of Astronomy, Yale University, PO Box 208101, New Haven, CT 06520-8101, USA \\ 3 Department of Physics and Astronomy, Michigan State University, East Lansing, MI 48824, USA \\ e-mail: zepf@pa.msu.edu \\ 4 European Southern Observatory, 85749 Garching bei München, Germany \\ e-mail: mkissler@eso.org \\ 5 Sternwarte der Universität Bonn, Auf dem Hügel 71, 53121 Bonn, Germany \\ e-mail: mhilker@astro.uni-bonn.de \\ ${ }^{6}$ Departamento de Astronomía y Astrofísica, P. Universidad Católica, Casilla 104, Santiago 22, Chile \\ e-mail: dante@astro.puc.cl \\ 7 Space Telescope Science Institute, 3700 San Martin Drive, Baltimore, MD 21218, USA \\ e-mail: goudfroo@stsci.edu
}

Received 18 February 2002 / Accepted 15 May 2002

\begin{abstract}
We combine near-infrared photometry obtained with the VLT/ISAAC instrument and archival HST/WFPC2 optical images to determine VIK magnitudes and colours of globular clusters in two early-type galaxies, NGC 3115 and NGC 4365. The combination of near-IR and optical photometry provides a way to lift the age-metallicity degeneracy. For NGC 3115 , the globular cluster colours reveal two major sub-populations, consistent with previous studies. By comparing the $V-I, V-K$ colours of the NGC 3115 globular clusters with stellar populations models, we find that the colour difference between the two $\gtrsim 10 \mathrm{Gyr}$ old major sub-populations is primarily due to a difference in metallicity. We find $\Delta[\mathrm{Fe} / \mathrm{H}]=1.0 \pm 0.3$ dex and the blue (metal-poor) and red (metal-rich) globular cluster sub-populations being coeval within 3 Gyr. In contrast to the NGC 3115 globular clusters, the globular cluster system in NGC 4365 exhibits a more complex age and metallicity structure. We find $a$ significant population of intermediate-age very metal-rich globular clusters along with an old population of both metal-rich and metal-poor clusters. Specifically, we observe a large population of globular clusters with red $V-K$ colours but intermediate $V-I$ colours, for which all current stellar population models give ages and metallicities in the range $\sim 2-8 \mathrm{Gyr}$ and $\sim 0.5 Z_{\odot}-3 Z_{\odot}$, respectively. After $10 \mathrm{Gyr}$ of passive evolution, the intermediate-age globular clusters in NGC 4365 will have colours which are consistent with the very metal-rich population of globular clusters in giant elliptical galaxies, such as M 87. Our results for both globular cluster systems are consistent with previous age and metallicity studies of the diffuse galactic light. In addition to the major globular cluster populations in NGC 3115 and NGC 4365 we report on the detection of objects with extremely red colours $(V-K \gtrsim 3.8 \mathrm{mag}$ ), whose nature could not ultimately be revealed with the present data.
\end{abstract}

Key words. galaxies: formation - galaxies: star clusters - galaxies: individual: NGC 3115, NGC 4365

\section{Introduction}

Globular cluster systems are useful tracers of galaxy evolution. They consist of bright clusters, which are made of stars that share the same age and chemical composition. Globular

Send offprint requests to: T. H. Puzia,

e-mail: puzia@usm.uni-muenchen.de

* Based on observations obtained at the European Southern Observatory, Chile (Observing Programme 63.N-0287).

$\star \star$ Based on observations made with the NASA/ESA Hubble Space Telescope, obtained from the data archive at the Space Telescope Institute. STSCI is operated by the association of Universities for Research in Astronomy, Inc. under the NASA contract NAS 5-26555. clusters can form during major merger events in galaxies (e.g. Holtzman et al. 1992; Whitmore et al. 1993; Whitmore \& Schweizer 1995; Schweizer et al. 1996, etc.), but we also observe the formation of massive star cluster in galaxies with a moderate star formation rate (e.g. O'Connell et al. 1994; Barth et al. 1995; O’Connell et al. 1995; Brandl et al. 1996; Larsen \& Richtler 1999; Hunter et al. 2000, etc.). In recent years the study of globular cluster systems revealed the presence of globular cluster sub-populations which must have formed in multiple formation epochs and/or mechanisms (see Ashman \& Zepf 1998; Kissler-Patig 2000; van den Bergh 2000; Harris 2001, for reviews). 
In practice, for distant galaxies, we observe integrated properties of their globular clusters, and would like to deduce their physical properties, in particular their age and metallicity. Unfortunately, even in systems where reddening can be taken as uniform, the optically observed parameters suffer from the well known age-metallicity degeneracy. Spectroscopy can overcome this infamous problem (Jones \& Worthey 1995; Worthey \& Ottaviani 1997; Vazdekis \& Arimoto 1999) to a large extent, but is, however, very time consuming to perform for hundreds of objects. Photometry still represents the most efficient way to study an entire globular cluster system.

There have been several attempts to solve this degeneracy for globular cluster systems in early-type galaxies which employed optical colours only (e.g. Kissler-Patig et al. 1997; Whitmore et al. 1997; Kissler-Patig et al. 1998; Kundu et al. 1999; Puzia et al. 1999) with various degrees of success. Previous studies used the optical colour difference and the turnover magnitude difference between the major globular cluster sub-populations to derive an age and metallicity difference. This method suffers from two main problems: 1) It is based on the assumption that the mass functions are similar for both sub-populations, so that luminosity differences directly reflect differences in the mass-to-light ratios of the individual populations. 2) The peak colour and turn-over magnitude differences are small and somewhat model dependent.

Alternatively one could derive mean age and metallicity differences between globular cluster sub-populations from colour-colour diagrams, which does not depend on the globular-cluster mass functions.

The combination of optical and near-infrared (near-IR) photometry can largely reduce the age-metallicity degeneracy. This is because optical to near-IR indices like $V-K$ are very sensitive to metallicity, but only have modest age sensitivity (Kissler-Patig 2000; Puzia et al. 2001; Kissler-Patig et al. 2002, hereafter Paper I). Physically, this technique works because the $V$-band samples mainly the light of stars near the turn-off, while the $K$-band is most sensitive to cooler stars on the giantbranch in old stellar populations (see e.g. Yi et al. 2001). While the turn-off is mostly affected by age, the giant branch is primarily sensitive to metallicity (e.g. Saviane et al. 2000). Thus, $V-I$ and $V-K$ are affected similarly by age, but $V-K$ is much more sensitive to metallicity (see Table 4 below). This allows the age-metallicity degeneracy to be lifted by determining the location of a simple stellar population in a plot of $V-I$ vs. $V-K$.

Such diagrams are powerful tools to study the age and the metallicity of globular-cluster populations. Including near-IR passbands to our optical data we can increase the sensitivity to metallicity differences by a factor of two or more (see Paper I, and SSP models of e.g. Bruzual \& Charlot 2000).

In this paper we study the globular cluster systems of NGC 3115 and NGC 4365. NGC 3115 is an isolated galaxy, located at the very tip of the southern extension of the Leo Group, with just one significant accompanying nucleated dwarf elliptical galaxy (Puzia et al. 2000a). It features a bimodal optical colour distribution of globular clusters (Kundu \& Whitmore 1998; Gebhardt \& Kissler-Patig 1999; Larsen et al. 2001).
Table 1. Basic information on observed galaxies. The references are (1): de Vaucouleurs et al. (1991), (2): Schlegel et al. (1998), (3): Buta $\&$ Williams (1995), (4): Frogel et al. (1978). (5): Tonry et al. (2001).

\begin{tabular}{lrrl}
\hline \hline Parameter & NGC 3115 & NGC 4365 & Ref. \\
\hline type & S0 & E3 & $(1)$ \\
RA $(\mathrm{J} 2000)$ & $10 \mathrm{~h} 05 \mathrm{~m} 14 \mathrm{~s}$ & $12 \mathrm{~h} 24 \mathrm{~m} 28 \mathrm{~s}$ & $(1)$ \\
DEC (J2000) & $-07^{\circ} 43^{\prime} 07^{\prime \prime}$ & $+07^{\circ} 19^{\prime} 03^{\prime \prime}$ & $(1)$ \\
$l$ & $247.78^{\circ}$ & $283.80^{\circ}$ & $(1)$ \\
$b$ & $36.78^{\circ}$ & $69.18^{\circ}$ & $(1)$ \\
$B_{\mathrm{T}, 0}$ & 9.74 & 10.49 & $(1)$ \\
$E_{B-V}$ & 0.047 & 0.021 & $(2)$ \\
$(B-V)_{\mathrm{o}}$ & 0.94 & 0.95 & $(1)$ \\
$(V-I)_{\mathrm{eff}, \mathrm{o}}$ & 1.25 & 1.25 & $(3)$ \\
$(V-K)_{\mathrm{eff}, \mathrm{o}}$ & $3.30 \pm 0.02$ & $3.29 \pm 0.1$ & $(4)$ \\
$(m-M)_{V}$ & $29.93 \pm 0.09$ & $31.55 \pm 0.17$ & $(5)$ \\
$M_{V}$ & -21.13 & -22.01 & $(1),(5)$ \\
\hline
\end{tabular}

NGC 4365 is a cluster giant elliptical galaxy located at the outer edge of the Virgo Cluster. Optical photometry of the globular cluster system revealed a broad but single-peak colour distribution with little evidence for bimodality (Forbes 1996; Gebhardt \& Kissler-Patig 1999; Larsen et al. 2001; Kundu \& Whitmore 2001a). Surma \& Bender (1995, see also Davies et al. 2001) detected a decoupled, counter-rotating core, which consists of a younger and more metal-rich stellar population than the rest of the galaxy. Such decoupled cores are believed to be created in major merger events. All relevant galaxy properties are summarized in Table 1.

The major goal of this paper is to derive ages and metallicities of globular clusters from a comparison of optical/nearIR colours with several SSP models. Putting this results into a larger context, including previous findings from galactic integrated light studies, we can further constrain formation scenarios for both galaxies.

The outline of this paper reads as follows. In Sect. 2 we describe the data reduction and calibration of our new near-IR VLT/ISAAC and the archival optical HST/WFPC2 data. The main results are presented in Sect. 3 followed by a discussion in Sect. 4. All major findings are summarized in Sect. 5.

\section{The photometric data}

\subsection{New VLT/ISAAC near-infrared data}

\subsubsection{Basic reduction}

Deep images in $K_{\mathrm{s}}{ }^{1}$ were obtained in service mode (program 63.N-0287) with the Near-Infrared Spectrometer And Array Camera (ISAAC, see Moorwood et al. 1998) attached to the Unit Telescope 1 (Antu) of the European Southern Observatory's Very Large Telescope (VLT). ISAAC is

1 The $K_{\mathrm{S}}(K$ short $)$ filter is a slightly modified $K$-band filter with the wavelength range $2.03-2.30 \mu \mathrm{m}$. Contrary to the $K$ filter the transmission curve drops before the CO-absorption (at $\sim 2.4 \mu \mathrm{m}$ ) band making the $K_{\mathrm{s}}$ filter insensitive to $\mathrm{CO}$ abundance variations. Moreover, the sky background is reduced relative to the standard $K$-band due to the short-wavelength cut-off. 
equipped with a Rockwell Hawaii $1024 \times 1024$ pixels $\mathrm{Hg}$ :Cd:Te array. The pixel scale is $0.147^{\prime \prime} /$ pixel and the field-of-view $2.5^{\prime} \times 2.5^{\prime}$ on the sky. In the following we will always refer to the $K_{\mathrm{s}}$ filter as $K$. The ISAAC field was oriented such as to match the WFPC2 field of view.

The NGC 3115 data were obtained during the nights of April 4th to 10th 1999 while the NGC 4365 data were taken during the nights of April 9th and 10th and additionally on the night of June 2nd 1999. The exposures were split into O-SSSSS-O sequences, where S was a dithered sky exposure comprising of 5 single exposures and $\mathrm{O}$ was a single on-target observation. Each sky image was exposed with a detector integration time (DIT) of $10 \mathrm{~s}$ and two such images were averaged in the read-out electronics (NDIT $=2$ ). Thus, the total integration time for a sky image is $20 \mathrm{~s}$. An object image has a total integration time (DIT $\times$ NDIT) of $10 \times 10=100 \mathrm{~s}$. Since the night-sky luminosity changes on short time scales the crucial part in each near-IR data reduction is the proper treatment of sky subtraction. Our observation pattern allowed us to create mean sky images, which have been cleaned of stars prior to combination to a mastersky image. The data of each night have been processed individually. From each raw object image the mastersky was subtracted giving a sky/bias-subtracted object image. Those sky/bias-subtracted images were divided by a normalized masterskyflat, which is provided by the ESO quality control group. Eventually, all images were aligned using 15-20 objects common to all frames and averaged to give the final cleaned object image. The $F W H M$ of the stellar PSF in the final $K$ band image is $\sim 0.6^{\prime \prime}$ for NGC 3115 and NGC 4365. The total exposure time for NGC 3115 is $15500 \mathrm{~s}$, and $9500 \mathrm{~s}$ for NGC 4365.

\subsubsection{Photometric calibration}

For the photometric calibration of the NGC 3115 and NGC 4365 data set 11 and 9 near-IR standard stars have been taken throughout the nights (Persson et al. 1998). Each standard star was imaged five times, each exposure having the star centered at a different position on the chip. Standard-star photometry was performed with the photometry tool SExtractor v2.1.6 (Bertin \& Arnouts 1996) in an aperture of 30 pixels of diameter $(7.4 \times$ seeing $)$. The corrections to an infinite-diameter aperture were found to be negligible in a subsequent curve-ofgrowth analysis. After applying a $\kappa-\sigma$ clipping to all the measurements outliers have been deleted leaving 40 data points for the calibration of each data set. Due to the lack of sufficient spread in airmass among the standard-star measurements, we use the airmass term determined in the standard calibration of the ISAAC instrument for April 1999. Assuming that the colour terms are negligible in the near-infrared ${ }^{2}$, we find the following

\footnotetext{
${ }^{2}$ The theoretical value for the $J-K_{\mathrm{s}}$ colour term was determined by the data quality control group of the Paranal observatory being close to zero (see ISAAC Data Reduction Guide 1.5). Since no $J$-band data of NGC 3115 and NGC 4365 are available and the optical and near-IR photometric systems of Johnson and Persson do not overlap, i.e. they have no stars in common, it is not possible to include a $V-K$ colour term into the calibration.
}

calibration relations for the photometric nights serving as reference

$$
\begin{aligned}
& K_{3115}=k_{\text {inst }}+23.56( \pm 0.018)-0.05( \pm 0.005) \chi \\
& K_{4365}=k_{\text {inst }}+23.75( \pm 0.015)-0.05( \pm 0.005) \chi
\end{aligned}
$$

where $K_{\text {galaxy }}$ is the calibrated magnitude, $k_{\text {inst }}$ is the instrumental magnitude normalised to $1 \mathrm{~s}$, and $\chi$ the airmass. The error of the zero points includes photometric errors of each single standard star measurement and the errors of the curve-of-growth analysis. The error of the airmass term is a estimate from the variations in airmass of all single exposures.

After correcting all nights to a common zero point (obtained from a reference photometric night) we traced the calibrated magnitude of two isolated bright stars over all nights. We find an upper-limit 1- $\sigma$ scatter being a few hundredths of a magnitude. Thus, we conservatively estimate the true photometric uncertainty, mainly driven by the strongly varying sky background, to be of the order of $\leq 0.03 \mathrm{mag}$.

Finally, all magnitudes were corrected for Galactic foreground reddening using the reddening values of Table 1 and the extinction curves of Cardelli et al. (1989). The corrections for NGC 3115 and NGC 4365 are $A_{K}=0.017$ mag and $A_{K}=0.008 \mathrm{mag}$.

\subsection{Archival HST/WFPC2 optical data}

NGC 3115 was observed with HST + WFPC2 under program GO.5512, with the PC centered on the nucleus of the galaxy. The total exposure times of the combined images are $3 \times 350 \mathrm{~s}$ in F555W, and $3 \times 350 \mathrm{~s}$ in F814W. NGC 4365 has been imaged with WFPC2 under program GO.5920 in F555W and F814W filters with $2200 \mathrm{~s}$ and $2300 \mathrm{~s}$ of total exposure time, respectively. The HST images were reduced and calibrated following for most parts the procedure as described in Puzia et al. (1999). Briefly recapitulating, all sub-exposures were matched by whole-pixel shifts and stacked using a cosmic-ray cleaning routine (crrej) within IRAF ${ }^{3}$. The object finding was performed on a master image which was created from all available exposures of galaxy to allow reliable cosmic-ray cleaning. None of the images suffers from crowding. Therefore, all magnitudes were measured with the SExtractor tool using a 8 and 4-pixel-diameter aperture for the WF and PC chip, respectively. Aperture corrections to the Holtzman standard aperture $\left(0.5^{\prime \prime}\right.$, see Holtzman et al. 1995) were determined in a curve-ofgrowth analysis. Only objects within the expected colour range for globular clusters were used (see Sect. 2.3). The correction terms can be found in Table 2. Instrumental magnitudes were then transformed to the Johnson $V$ and $I$ magnitudes according to the prescription given in Holtzman et al. All magnitudes were reddening corrected using the reddening values as listed in Table 1. Together with the extinction curves of Cardelli et al. (1989) we obtain $A_{V}=0.157$ and $A_{I}=0.092$ in the direction of NGC 3115 and $A_{V}=0.070$ and $A_{I}=0.041$ for NGC 4365 .

\footnotetext{
3 IRAF is distributed by the National Optical Astronomy Observatories, which are operated by the Association of Universities for Research in Astronomy, Inc., under cooperative agreement with the National Science Foundation.
} 
Table 2. Aperture corrections for WFPC 2 photometry. The given terms refer to the correction from an 8/4-pixel-diameter aperture (PC/WF chips) to the standard Holtzman 0.5" aperture. \# indicates the number of objects which were used to determine the correction.

\begin{tabular}{ccccccr}
\hline \hline & & PC1 & WF2 & WF3 & WF4 & $\langle$ WF $\rangle$ \\
\hline NGC 3115 & V & -0.152 & -0.296 & -0.385 & -0.323 & -0.323 \\
& I & -0.236 & -0.370 & -0.412 & -0.330 & -0.362 \\
& $\#$ & 6 & 25 & 11 & 24 & \\
\hline NGC 4365 & V & -0.065 & -0.134 & -0.159 & -0.127 & -0.141 \\
& I & -0.133 & -0.205 & -0.242 & -0.219 & -0.225 \\
& $\#$ & 28 & 66 & 39 & 67 & \\
\hline
\end{tabular}

In the case of NGC 3115 excellent agreement was found between our data set and the previous studies of Kundu \& Whitmore (1998) and Gebhardt \& Kissler-Patig (1999). The systematic offset between the Kundu \& Whitmore photometry and ours is $0.04 \pm 0.11 \mathrm{mag}$ in $V_{\mathrm{F} 555 \mathrm{~W}}$ and $-0.01 \pm 0.11$ in $I_{\mathrm{F} 814 \mathrm{~W}}$. We find also good agreement for NGC 4365 between the photometry of Gebhardt \& Kissler-Patig (1999) and our data (but see also Puzia et al. 2000b). In $V_{\mathrm{F} 555 \mathrm{~W}}$ we measure an offset $0.04 \pm 0.07 \mathrm{mag}$ and $0.07 \pm 0.05 \mathrm{mag}$ for the $I_{\mathrm{F} 814 \mathrm{~W}}$ filter.

\subsection{Selection of globular cluster candidates}

For object selection the near-infrared ISAAC data were combined with the archival optical HST data. Note that the WFPC2 field size of $2.66^{\prime} \times 2.66^{\prime}$ is well matched to the ISAAC field of view $\left(2.5^{\prime} \times 2.5^{\prime}\right)$. After transformation of HST coordinates to the ISAAC frame we required the matches being better than $0.74^{\prime \prime}(\sim$ seeing). Before selecting for likely GCs, the matched lists contain 110 and 186 objects with VIK photometry for NGC 3115 and NGC 4365, respectively. To get rid of extended sources and noisy detections, we selected GC with cuts in $F W H M_{\mathrm{WFPC} 2}$ and photometric error, as measured on the optical HST data. Figure 1 illustrates the constraints which define our globular cluster candidates. For NGC 3115, all objects with a $F W H M_{\text {WFPC2 }}$ larger than $0.4^{\prime \prime}$ and for NGC 4365 larger than $0.2^{\prime \prime}$ were rejected. Both $F W H M_{\mathrm{WFPC} 2}$ values roughly correspond to $20 \mathrm{pc}$ at the distance of $9.7 \mathrm{Mpc}$ and $20.4 \mathrm{Mpc}$ for NGC 3115 and NGC 4365, respectively (see Tonry et al. 2001). To determine the real sizes of clusters which are rejected by the $F W H M_{\mathrm{WFPC} 2}$ cut we convolve the stellar PSF in each image with a series of King profiles (King 1962) with a mean concentration parameter $c=\log \left(r_{\mathrm{t}} / r_{\mathrm{c}}\right)=1.55$, which was determined from the Milky Way globular cluster system (Harris 1996). The tests yield a maximum allowed core radius $r_{\mathrm{c}} \approx 5.6 \mathrm{pc}$ and $4.9 \mathrm{pc}$, for NGC 3115 and NGC 4365 globular clusters, to be retained in the sample. An inspection of the core radii of Milky Way globular clusters shows that $82 \%$ and $79 \%$ have core radii smaller than the two respective maximum allowed sizes mentioned above. Unless the size distribution of NGC 3115 and NGC 4365 globular clusters differs significantly, which seems unlikely given the similarity in sizes observed for many other early-type galaxies (e.g. Kundu \& Whitmore 1998; Larsen et al. 2001), we will miss only the
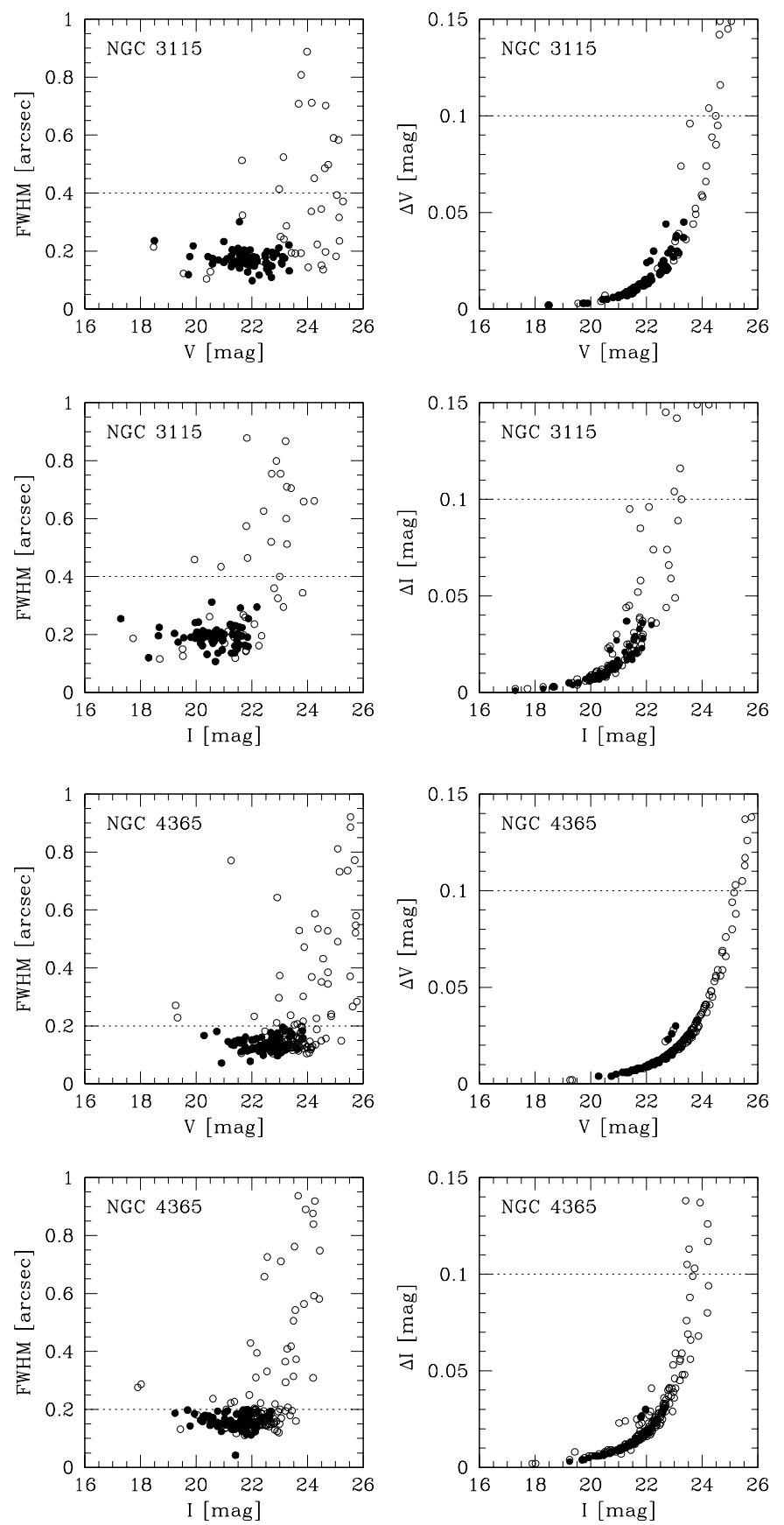

Fig. 1. Selection parameter as a function of $V_{\mathrm{F} 555 \mathrm{~W}}$ and $I_{F 814 W}$ magnitude. Plotted here are the full width at half maximum (FWHM) of the point-spread function (PSF) and the photometric error, as derived on HST images. Data points which were rejected during the selection are marked as open circles. Dotted lines indicated the selection boundaries.

extremely large globular clusters by applying the $F W H M_{\mathrm{WFPC} 2}$ cuts.

Furthermore, only objects with photometric errors in the HST photometry of less than $0.1 \mathrm{mag}$ in $V$ and $I$ were retained. After the selection procedure the NGC 3115 list contains 87 candidates while the NGC 4365 list comprises 136 globular cluster candidates ${ }^{4}$.

${ }^{4}$ The final lists are available in electronic form from THP. 
Background contamination is unlikely to make a significant contribution to the selected samples. Puzia et al. (1999) have shown that with similar selection criteria the background contamination is less than $5 \%$.

\subsection{Completeness}

The HST data for NGC 3115 is complete down to $V_{\mathrm{F} 555 \mathrm{~W}} \lesssim 26$ and $I_{\mathrm{F} 814 \mathrm{~W}} \lesssim 25$. For NGC 4365 the photometry reaches $V_{\mathrm{F} 555 \mathrm{~W}} \lesssim 26.5$ and $I_{\mathrm{F} 814 \mathrm{~W}} \lesssim 25.5$. Clearly, the limiting photometric passband is the $K$ filter for both our galaxies and it will define the completeness of the data. Artificial star experiments with 1000 cycles, each including 100 artificial objects into the $K$ images, yield a limiting magnitude (50\% completeness) of $K \lesssim 20.0$ and $K \lesssim 20.25$ for NGC 3115 and NGC 4365, respectively.

Despite the much longer exposure time for NGC 3115 $(15500 \mathrm{~s})$ the final $K$-band limiting magnitude is about the same as for NGC 4365 (9500 s). Purely from the ratio of exposure times one would expect the NGC 3115 photometry being 0.53 mag deeper than for NGC 4365 . However, we find the NGC 4365 photometry is 0.25 mag deeper which adds up to a total difference of $\sim 0.78 \mathrm{mag}$ or a factor $\sim 1.4$ in $S / N$.

\subsubsection{Surface brightness fluctuations}

This discrepancy cannot be explained by a higher noise due to a difference in surface brightness between the halos of NGC 3115 and NGC 4365, since they are measured to be the same within $0.1 \mathrm{mag} / \operatorname{arcsec}^{2}$. However, a factor contributing to the lower detection limit in the NGC 3115 field are the larger $K$-band surface brightness fluctuations (SBFs) (Tonry \& Schneider 1988):

In addition to the Poisson noise of the flux, each resolutions element contains SBFs which are the Poissonian fluctuations of the sampled number of stars. If the resolution element samples $n$ stars of the mean flux $\bar{F}$ then the fluctuation of the flux will be $\sigma_{F}=\bar{F} \sqrt{n}$. Hence, putting a galaxy twice as far away from the observer will keep its surface brightness constant (one resolution element samples now 4 times more stars which have 4 times lower flux) but lower the SBFs by a factor 2 . The SBF amplitude is proportional to the distance $\sigma_{F} \propto D^{-1}$. Both NGC 3115 and NGC 4365 have roughly the same $K$-band surface brightness, $\mu_{3115}=12.5$ and $\mu_{4365}=12.6 \mathrm{mag} \mathrm{arcsec}^{-2}$, at radii including the majority of our globular cluster candidates. However, since NGC 3115 is roughly two times closer than NGC 4365 (Tonry et al. 2001), the ratio of SBF amplitudes, $\sigma_{F} \propto S / N$, will be $\sim 2$ (compared to $\sim 1.4$ measured). Thus, relaxing the implicit assumption of equal stellar populations, the difference in the SBF amplitude can explain the difference in the limiting magnitudes.

\subsubsection{Colour completeness}

Regardless of the limiting magnitude, our data samples are biased toward red globular clusters. That is, the red (metal-rich) sub-population is more complete than the blue (metal-poor) one at a given magnitude threshold (see Fig. 3). To quantify the biasing factor we assume that the globular cluster luminosity functions (GCLFs) of both sub-populations have the same shape (same mass function) and the same dispersion (same slopes of the mass function). Both globular cluster subpopulations are assumed to have the same M/L. Previous studies of the globular clusters systems of elliptical and S0 galaxies suggest that this assumption is a reasonable initial approximation, although in detail there is some evidence for modest differences in the $\mathrm{M} / \mathrm{L}$ between the metal-poor and metal-rich subpopulations of well-studied cluster systems (see Puzia et al. 1999; Kundu et al. 1999, for a discussion on NGC 4472 and M 87).

The turn-over magnitude of the NGC $3115 \mathrm{GCLF}$ is $V_{\mathrm{TO}}=$ $22.6 \pm 0.2$ for both blue and red cluster candidates (Larsen et al. 2001). Concerning the mean $V-K$ colour for the blue $(2.3 \pm 0.1 \mathrm{mag}$, see Sect. 3.1 and Table 3$)$ and for the red clusters $(3.0 \pm 0.1 \mathrm{mag})$ the expected turn-over magnitudes in $K$ are $K_{\mathrm{TO} \text {,blue }} \approx 20.3$ and $K_{\mathrm{TO} \text {,red }} \approx 19.6 \mathrm{mag}$. According to the completeness limit of the NGC 3115 data $\left(K_{\text {lim }} \approx 20.0 \mathrm{mag}\right)$ and assuming a GCLF dispersion $\sigma_{\mathrm{GCLF}}=1.3$, we sample $41 \%$ of the blue and $62 \%$ of the red sub-population. The biasing factor for the NGC 3115 globular cluster system is thus $\sim 1.5$. That is, we detect $\sim 1.5$ times more red clusters than blue ones. In the case of NGC 4365 we detect $13 \%$ of blue and $23 \%$ of red clusters assuming $V_{\mathrm{TO}}=24.37 \pm 0.16$ mag along with $\sigma_{\mathrm{GCLF}}=1.22 \mathrm{mag}$ (Larsen et al. 2001) and $V-K=2.76$ and $3.23 \mathrm{mag}$ for blue and red clusters, respectively. The biasing factor is 1.8 . If we assume a single peak colour distribution with a mean $V-K \approx 2.95$ (see Sect. 3.1) and a turn-over magnitude independent of colour we sample $17 \%$ of the GCLF in NGC 4365.

\subsubsection{Spatial completeness}

Both our globular cluster samples are spatially incomplete (see Fig. 2). Moreover, the different spatial distribution of blue and red globular clusters also influences the completeness. In NGC 3115 we sample the inner $4 \mathrm{kpc}$ of the globular cluster system. The spatial coverage of NGC 4365 globular clusters is more complete. Our field of view covers clusters with galactocentric distances up to $\sim 10 \mathrm{kpc}$. For NGC 3115 , Kundu \& Whitmore (1998) report that the red globular clusters follow the galactic light profile while the blue clusters rather reside in the halo. Thus, independent of completeness biases, both samples have to be considered as spatially biased in favour of red globular clusters. The spatial distribution of globular cluster candidates is shown in Fig. 2, along with the location of the WFPC 2 and ISAAC apertures.

\section{Results}

\subsection{Colour distributions and colour-magnitude diagrams}

We use the KMM code (Ashman et al. 1994) to test for bimodality of the colour distributions. The code calculates the likelihood $P_{\mathrm{KMM}}$ whether or not a two-Gaussian fit is 

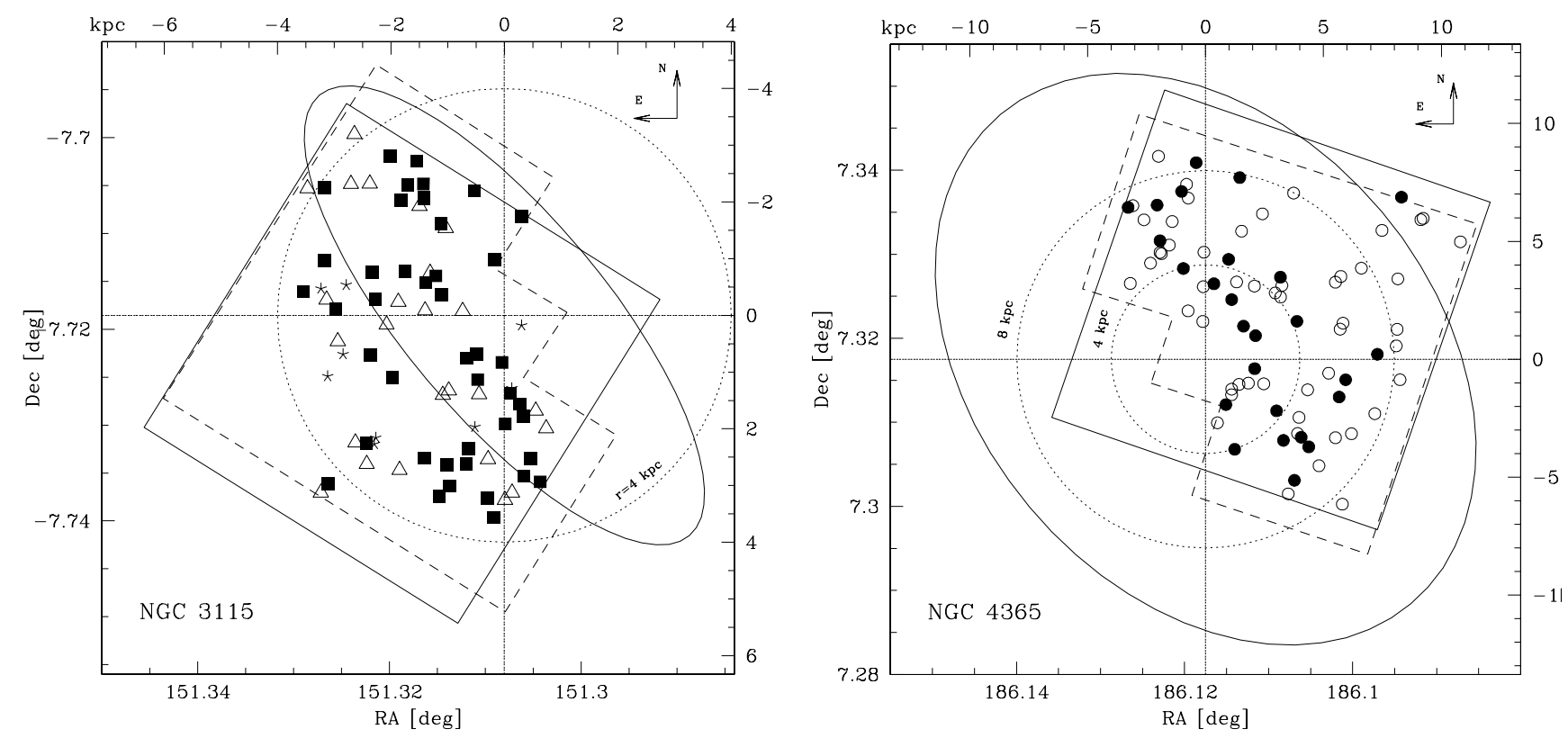

Fig. 2. Field of view for the NGC 3115 (left panel) and NGC 4365 (right panel) data on the sky. The solid square shows the effective aperture of the ISAAC data. The HST aperture is indicated as a dashed polygon. Solid ellipses indicate the area encompassing half of the integrated light of the galaxy. For NGC 3115, we split the sample at $V-I=1.05$ and plot the blue sub-population as open triangles and the red clusters as filled squares. Stars mark the position of extremely-red objects in the colour range $3.7<V-K<4.3$, which meet our completeness but not the size selection criteria (all extremely-red objects lie above the dashed line in Fig. 3). For NGC 4365, solid circles show the positions of globular clusters with intermediate ages, whereas the remainder globular cluster candidates are marked by open circles.

preferred over a single-Gaussian fit and determines the positions of the modes. Higher $P_{\text {KMM }}$ values indicate that a singlepeak distribution is more likely. In addition to the cuts in $F W H M_{\mathrm{WFPC} 2}$ and photometric error (see Sect. 2.3), we select globular cluster candidates by their $V-K$ colour in the range $1.7<(V-K)<3.7$ and cut the samples at the limiting $K$ magnitude (NGC 3115: $K \lesssim 20.0$, and NGC 4365: $K \lesssim 20.25$ ). According to simple stellar population models (see e.g. Fig. 6) this colour range selects globular clusters with metallicities $-2.5 \lesssim[\mathrm{Fe} / \mathrm{H}] \lessgtr+0.4$ dex and with a wide range of ages. The input lists contain now 69 and 78 objects for NGC 3115 and NGC 4365, respectively.

KMM returns for NGC 3115 a likelihood of $P_{\mathrm{KMM}}=0.53$ with peaks at $V-K=2.31 \pm 0.06$ and $3.01 \pm 0.04$. Former studies found a clear bimodality in the $V-I$ colour distribution of globular clusters in NGC 3115. Kundu \& Whitmore (1998) find peaks at $V-I=0.96$ and 1.17 and the most likely separation of the two sub-populations being at $(V-I)_{\text {dip }}=1.06$. Gebhardt \& Kissler-Patig (1999), who first calculated the likelihood of a dip in a supposedly bimodal colour distribution, give $(V-I)_{\text {mean }}=1.03 \pm 0.03$ with a dip likelihood $P_{\text {dip }}=0.71$. Larsen et al. (2001) find peaks ${ }^{5}$ at $V-I=0.92 \pm 0.03$ and $1.15 \pm 0.03$ and a dip likelihood $P_{\mathrm{dip}}=0.96$.

As for NGC 4365, KMM gives $P_{\mathrm{KMM}}=0.94$ with peaks at $V-K=2.76 \pm 0.04$ and $3.23 \pm 0.04$. In NGC 4365 Gebhardt \& Kissler-Patig (1999) find a hint for bimodality in their $V-I$ colour distribution. The mean colour and the bimodality significance of their distribution are $(V-I)_{\text {mean }}=1.09 \pm 0.02$ and

\footnotetext{
5 The errors of the colour peaks were kindly provided by Søren Larsen.
}

$P_{\text {dip }}=0.76$. Larsen et al. (2001) measure $V-I=0.98 \pm 0.03$ and $1.19 \pm 0.03$ as peak colours and a dip likelihood $P_{\text {dip }}=0.033$. These results show that there are some galaxies for which extant colour distributions do not give a clear result when statistical tests are applied to search for bimodality. No clear multimodality is detectable in either colour distribution (for $V-I$ see Gebhardt \& Kissler-Patig 1999; Larsen et al. 2001; Kundu \& Whitmore 2001a). The globular clusters show a continuous colour distribution.

The errors of $V-K$ peak colours in our KMM analysis have been determined by varying the input parameter of the KMM code, which are the initial colour for both peaks, their covariance, and whether or not the two peaks have the same dispersion. All values are summarized in Table 3 . Note, that the driving parameters for detecting bimodalities with KMM are the peak locations, the dispersions, and the sample size. With smaller samples the $P_{\text {KMM }}$ parameter becomes less meaningful (Ashman et al. 1994). The bimodality test shows that only the colour distribution of NGC 3115 is likely to be not unimodal. Contrary, NGC 4365 has a globular cluster colour distribution, which is well represented by a single Gaussian. See Fig. 3 for $(V-K)$ histograms.

A metallicity calibration of $V-K$ using Milky Way and M 31 globular clusters with $E_{B-V}<0.27$ is derived in Paper I

$[\mathrm{Fe} / \mathrm{H}]=-5.52( \pm 0.26)+1.82( \pm 0.11) \cdot(V-K)$

with a rms of $0.29 \mathrm{dex}$. With this calibration we calculate metallicities from peak colours which were obtained in the KMM analysis. The globular cluster sub-populations in NGC 3115 have then metallicities $[\mathrm{Fe} / \mathrm{H}]=-1.32 \pm 0.11$ and $-0.04 \pm$ 0.07 dex. Transforming the KMM peaks of the NGC 4365 
Table 3. KMM mean colours of the globular cluster sub-populations in NGC 3115 and NGC 4365.

\begin{tabular}{llcc}
\hline \hline & & $V-K$ & $V-I^{\mathrm{a}}$ \\
\hline \multirow{2}{*}{ NGC 3115 } & blue & $2.31 \pm 0.06$ & $0.922 \pm 0.03$ \\
& red & $3.01 \pm 0.04$ & $1.153 \pm 0.03$ \\
\hline \multirow{2}{*}{ NGC 4365 } & blue & $2.76 \pm 0.04$ & $0.981 \pm 0.03$ \\
& red & $3.23 \pm 0.04$ & $1.185 \pm 0.03$ \\
\hline
\end{tabular}

a Values for the blue and red sub-populations adopted from Larsen et al. (2001).

system yields $[\mathrm{Fe} / \mathrm{H}]=-0.50 \pm 0.07$ and $0.36 \pm 0.07$ dex. The median $V-K$ colours of the globular cluster systems of NGC 3115 and NGC 4365 are equivalent to $[\mathrm{Fe} / \mathrm{H}]=$ $-0.15 \pm 0.07$ and $-0.33 \pm 0.05 \mathrm{dex}$, respectively. The errors include the uncertainty in the peak colours only. Note, that the calibration was established with Milky Way and M 31 globular clusters, which have very few clusters at higher metallicities (i.e. at $[\mathrm{Fe} / \mathrm{H}] \gtrsim-0.4 \mathrm{dex}$ ). Consequently, all derived values with metallicities around solar and higher may suffer from uncertainties due to extrapolations.

In Fig. 3 (bottom panels) we show $V$ vs. $V-K$ colourmagnitude diagrams (CMDs). Together with the $V-K$ histograms in Fig. 3 (top panels) the CMDs of NGC 3115 and NGC 4365 show no clear multi-modality due to the limited sample size. The dashed lines show the $50 \%$ completeness limits and make the selective completeness obvious.

The CMDs also show the data which were rejected by our object selection criteria $\left(F W H M_{\mathrm{WFPC} 2}\right.$, photometric error, and $V-K)$, which are shown as open circles. For NGC 3115, this includes two bright and blue likely foreground stars. Using the galactic stellar population model ${ }^{6}$ of Robin et al. (1996), we expect zero stars. In the case of NGC 4365 we find no blue contaminating objects, in agreement with the model predictions.

\subsection{Extremely red objects}

At the red end of the NGC 3115 and NGC 4365 CMD (Fig. 3) we find a dozen objects with $V-K \approx 4.0 \mathrm{mag}$, which were rejected by the $F W H M_{\mathrm{WFPC} 2}$, completeness, and photometricerror cuts. These objects show no significant clustering and are evenly distributed on the sky. Some of them show an extended PSF and their $F W H M_{\mathrm{WFPC} 2}$ values exceed our $F W H M$ threshold making them likely background galaxies. The nature of the remaining very red point sources (indicated by squares in Fig. 4), not rejected by these selection criteria but only by the $V-K$ colour cut, is less clear. We discuss in turn some possibilities of their nature:

Foreground stars: In the colour range $3.5 \lesssim V-K \lesssim$ $4.5 \mathrm{mag}$ and the magnitude range $22.0 \lesssim V \lesssim 24.5 \mathrm{mag}$ we expect two stars in the ISAAC field of view $\left(6.25 \operatorname{arcmin}^{2}\right)$,

\footnotetext{
6 The model predictions were calculated using the code at www . obs-besancon . fr/www/modele/modele_ang.html
}

according to the model of Robin et al. (1996). The effective field of view is even smaller due to the incomplete overlap between the ISAAC and WFPC2 field. That is, significant stellar contamination at this red colours and faint magnitudes can be considered as unlikely.

Fuzzy red globular clusters: In optical colours the extremely-red objects are clustered around $V-I \approx 1.4$ and 2.2 mag. Counterparts of the redder objects could be the recently detected population of extremely-red objects around NGC 1023, with typical colours $V-I \approx 2.2$, which appear to be more extended than the other globular cluster candidates (Larsen \& Brodie 2000). Using the resolution power of HST, we measure the $\Delta(0.5-3)$ parameter (which is simply the magnitude difference between the flux within two different aperture sizes; see e.g. Puzia et al. 1999) to study relative sizes of our globular cluster candidates. Figure 4 shows the relative size distribution of globular cluster candidates and all other objects of our samples. The wide-field chips of WFPC2 on-board HST resolve objects down to 4.5 and $9.6 \mathrm{pc}$ radius at the distance of NGC 3115 and NGC 4365, respectively. Red objects, indicated by an open square (in Fig. 4), were rejected by the $V-K$ colour selection only.

Intermediate-age globular clusters: We point out that the presence of carbon-star dominated intermediate-age globular clusters, like they are found in the LMC and SMC (Olszewski et al. 1996), is unlikely to explain the extremely red colours. The strong IR flux of such AGB stars, yields red $V-K$ colours but leaves the $V-I$ colour relatively unchanged. The $V-I$ colours of the extremely-red objects are too red to be matched by any simple stellar population at low redshift, regardless of its age and AGB contribution.

Background galaxies: Unresolved background galaxies can, however, also mimic a stellar PSF. In the case of our very red objects, we might deal with the blue end of the known population of Extremely Red Objects (EROs), identified as redshifted and/or obscured galaxies at $z>0.85$ (e.g. Liu et al. 2000; Daddi et al. 2001). The colour definition of EROs varies in the literature and is typically around $R-K \gtrsim 4.5-6$ mag. The surface density of EROs is a function of colour and limiting magnitude, and increases from $0.07 \mathrm{arcmin}^{-2}$ for $R-K \geq 6 \mathrm{mag}$ to $0.49 \mathrm{arcmin}^{-2}$ for $R-K \geq 5 \mathrm{mag}$ for a sample with $K_{\text {lim }} \leq 19.0 \mathrm{mag}$ (Daddi et al. 2001). In our field of view, we expect 0.4 and 3.1 EROs, respectively, down to $K=19$ mag. Our objects have similar colours as the bluest EROs of which the distribution on the sky is inhomogeneous. Hence, the number of very red objects in our field of view (two at $K \sim 19 \mathrm{mag}$ ) is consistent with the surface density of EROs. The red end of the NGC 4365 CMD shows also hints of an extremely-red population of objects fainter than the photometric limit. All, except two sources in this colour regime are rejected by the $F W H M_{\mathrm{WFPC} 2}$ selection and the completeness limit.

In conclusion, the nature of our extremely-red objects cannot be definitely clarified from the photometric data only. Spectroscopy is necessary to distinguish between cool Mtype foreground stars, obscured background galaxies, or (unobscured) extremely metal-rich young globular clusters. 

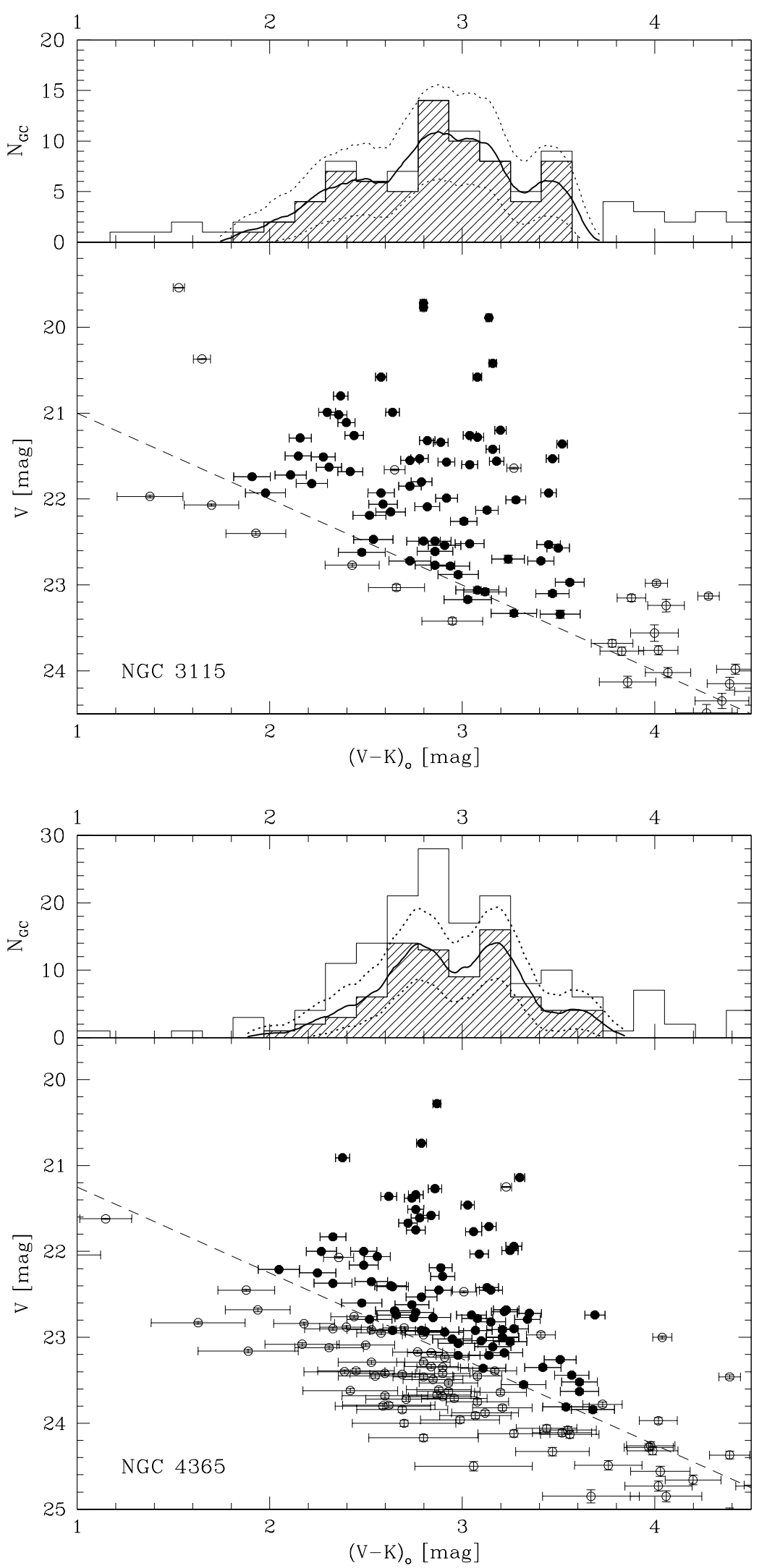

Fig. 3. $V$ vs. $V-K$ colour-magnitude diagrams for NGC 3115 (upper panel) and NGC 4365 (lower panel). The bottom of each panel shows the CMD of the associated globular cluster system. Filled symbols are selected GC while open symbols indicate data, which were rejected by our selection criteria (i.e., $F W H M_{\mathrm{WFPC} 2}$, photometric error, and colour). The dashed line shows the $50 \%$ completeness limit of the photometry as constrained by the near-IR photometry. The errors include photometric errors only. The top of each panel shows the $V-K$ histogram of all detected sources (solid histogram) and the selected GC sample (shaded histogram). A probability-density estimate (Silverman 1986; Puzia et al. 2000a) of the selected unbinned data is indicated (thick line) along with its $1 \sigma$ uncertainty limits (dotted lines). 


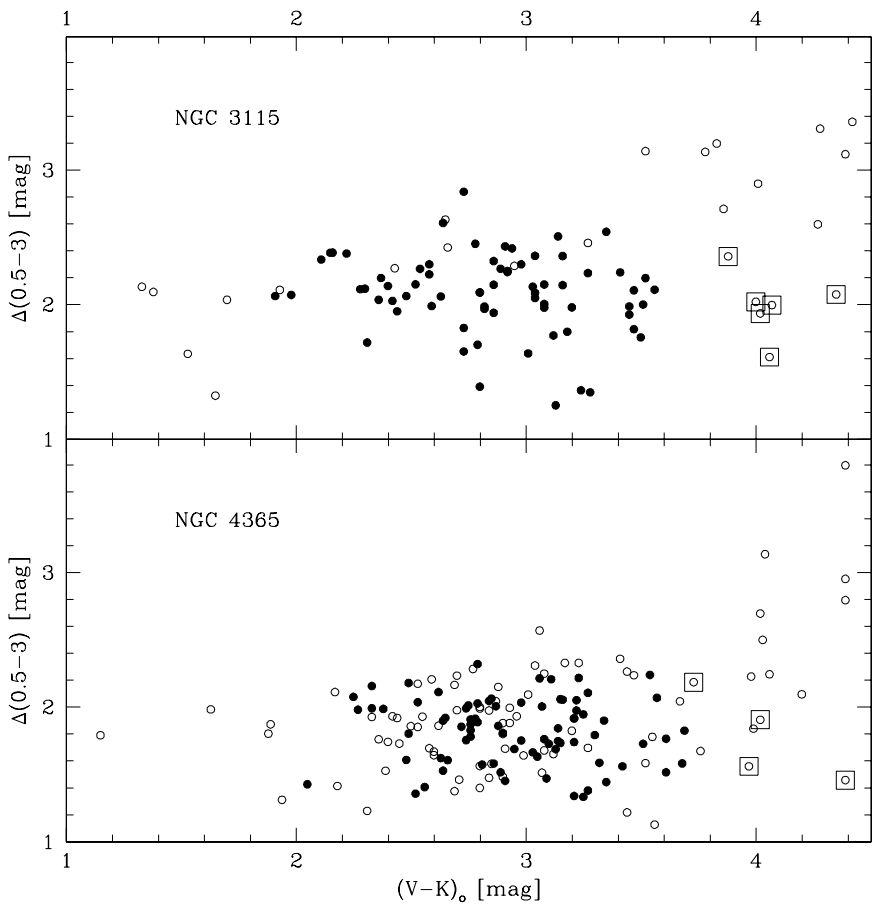

Fig. 4. Relative object sizes as a function of $V-K$ colour for NGC 3115 (top panel) and NGC 4365 (lower panel). Solid points show the data after application of the selection criteria $\left(F W H M_{\mathrm{WFPC} 2}\right.$, photometric error, and $V-K$ colour) while open circles show all data. Open squares show data with red colours which would pass the selection criteria if the $V-K$ selection were dropped (i.e. point-like very red objects). Note, that there is no trend of size vs. colour seen in either galaxy, contrary to the results of Larsen et al. who find that red globular clusters in near-by elliptical galaxies are on average $20 \%$ smaller than blue ones. However, this correlation might be camouflaged by the colour bias of our sample.

\subsection{Colour-colour diagrams}

With the VIK data in hand optical/near-IR colour-colour diagrams, such as $V-I$ vs. $V-K$, can be constructed.

In the following we compare globular cluster colours to different simple stellar population (SSP) models and determine the mean age and metallicity of the major sub-populations. We use the models of Vazdekis (1999), Kurth et al. (1999), Bruzual \& Charlot (2000), and Maraston (2001). Absolute calibrations of SSP models are nowadays still uncertain. However, the model-to-model differences of relative values are small. This is particularly true for the age and metallicity ranges covered by Milky Way and M 31 globular clusters which serve as model calibrators. Figure 5 demonstrates model-to-model variations for a stellar population of $12 \mathrm{Gyr}$ with a metallicity range $-1.7 \lesssim[\mathrm{Fe} / \mathrm{H}] \lesssim+0.4 \mathrm{dex}$. Also plotted are the globular clusters of M 31 and the Milky Way (Harris 1996; Barmby et al. 2000; Aaronson \& Malkan in prep.). The plot shows that at a fixed age, metallicity increases along the track towards redder colours. Note, that there is very few data for M 31 or Galactic globular clusters in the solar-metallicity regime. Tracks of younger ages fall generally below (i.e., have bluer $V-I$ colours than) older tracks and the colour differences between tracks increases with younger ages. For old ages the

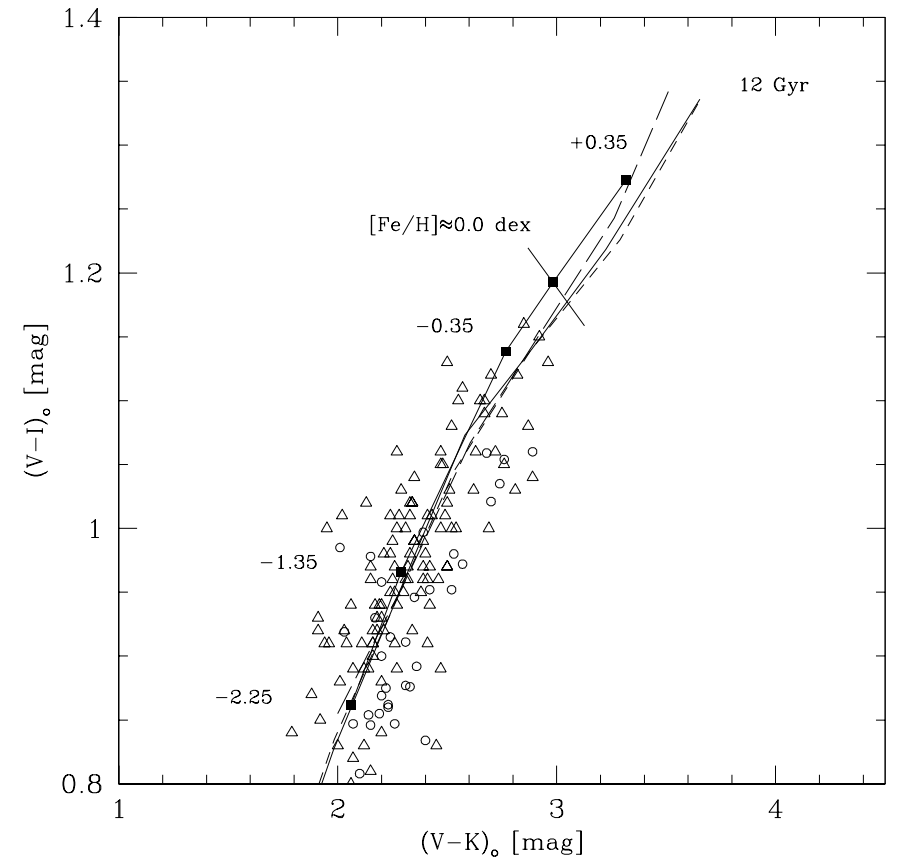

Fig. 5. Variations of model predictions for a 12 Gyr old simple stellar population in the metallicity range $[\mathrm{Fe} / \mathrm{H}]=-2.25$ to +0.35 dex. The lines indicate the predictions of Maraston (2001) (solid), Kurth et al. (1999) (short-dashed), Bruzual \& Charlot (2000) (dotted), and Vazdekis (1999) (long-dashed). The metallicity on the iso-age line of Maraston (2001) is labeled and indicated by filled squares. Open triangles are globular clusters of M 31 (Barmby et al. 2000) while open circles mark the colours of Milky Way globulars (Aaronson \& Malkan, in prep.). The $(V-I)$ data was taken from Harris (1996).

separation of tracks shrinks to values comparable with photometric errors which diminishes the ability to distinguish between ages.

\subsubsection{NGC 3115}

The colour-colour plot for NGC 3115 globular clusters in Fig. 6 show selected data with their photometric errors. Large squares show the mean colours of the two sub-populations as determined in the KMM analysis. Their error bars indicate the error of the mean as defined by $\sigma_{\text {mean }}=\sigma / \sqrt{N}$. As a guidance for the eye, iso-age and iso-metallicity lines taken from the Bruzual \& Charlot (2000) SSP model are overplotted. Dotted lines show iso-age tracks for 1 to $15 \mathrm{Gyr}$ populations with metallicities $-1.7 \leq[\mathrm{Fe} / \mathrm{H}] \leq+0.4 \mathrm{dex}$.

Figure 6 reveals two main results. First, there is a broad spread in metallicity with evidence for bimodality in the NGC 3115 cluster system. The globular cluster population can be split at around $V-I \approx 1.05$ and $V-K \approx 2.6$ into two major sub-populations. In general, the colour-colour diagram helps to pick out the multi-modality which is not evident in $V-I$ or $V-K$ colour distributions alone. The second result is that the two globular cluster sub-populations have both old ages with a small or a negligible age difference. Overall, the whole system seems to be dominated by old globular clusters. The colour difference is, therefore, largely due to a metallicity difference. 

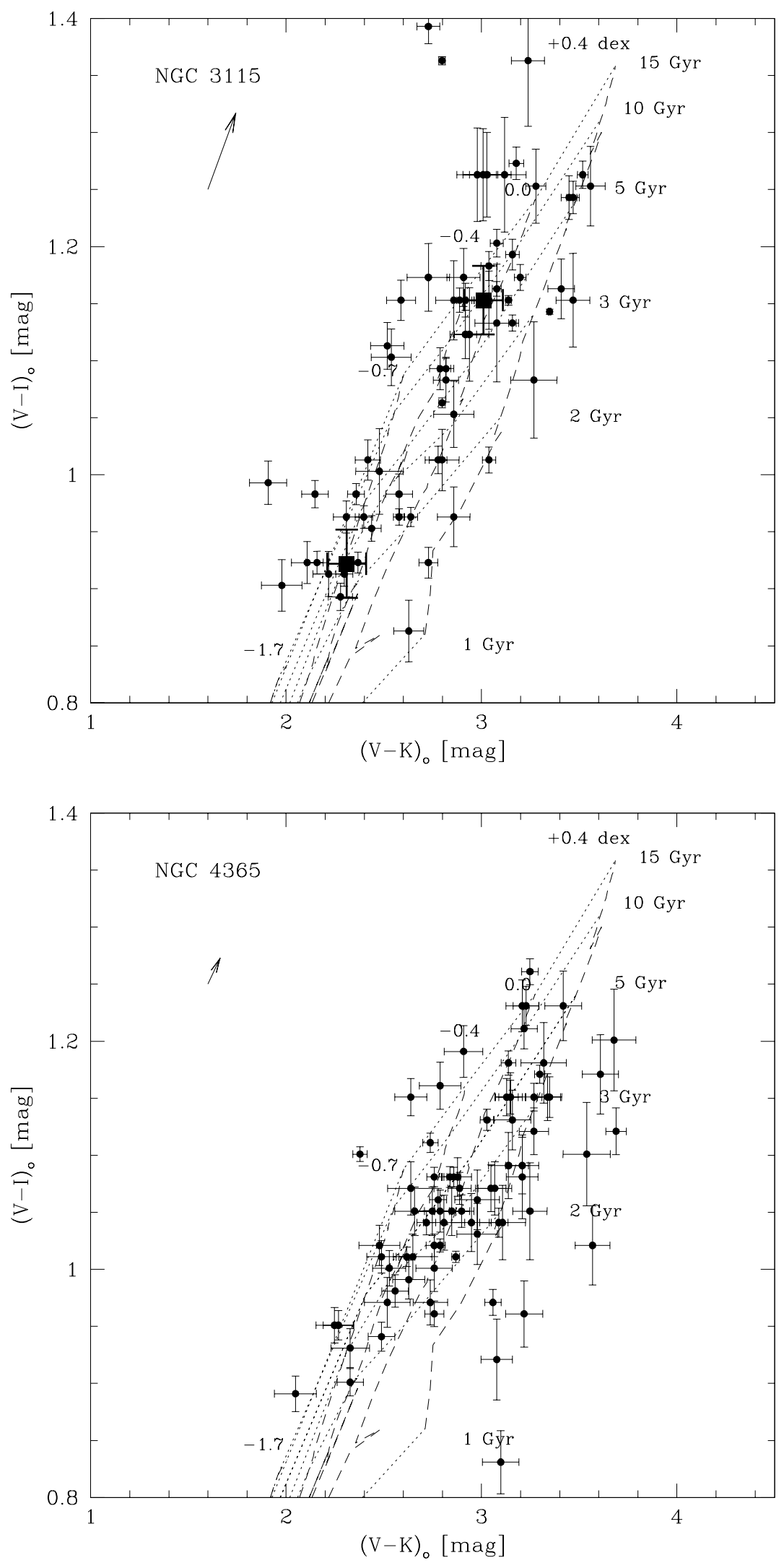

Fig. 6. $V-I$ vs. $V-K$ colour-colour diagrams for NGC 3115 and NGC 4365. All data are reddening corrected according to Sect. 2.1.2 and 2.2. For comparison, we show six isochrones for simple stellar populations of 15, 10, 5, 3, 2, and 1 Gyr (dotted lines from red to blue $V-I$ colours). The dashed lines represent iso-metallicity tracks changing from the lower left to the upper right from $[\mathrm{Fe} / \mathrm{H}]=-1.7$ to +0.4 dex. The SSP model used here was adopted from Bruzual \& Charlot (2000). For NGC 3115, the large squares mark the mean colour of the modes as determined by KMM code (see Sect. 3.1). Arrows in the upper left corner of each panel indicate the applied extinction correction and the direction of reddening. 
To quantify the age and metallicity difference between both globular cluster sub-populations, we compare the difference in their $V-I$ and $V-K$ colours to several SSP model predictions. We recall again that our data sample is biased towards red globular clusters, i.e., we detect 1.5 times more red clusters in the NGC 3115 system than blue ones (see Sect. 2.4). The blue peak of the $V-K$ colour distribution might, therefore, vary as one goes deeper with the $K$ band photometry, adding more blue globular clusters to the sample.

Figure 7 shows the mean colours of the two subpopulations overplotted on SSP model grids. From the model of Maraston (2001) we derive an age difference between the two sub-populations of $\Delta t=\left(t_{\text {blue }}-t_{\text {red }}\right)=2 \pm 4$ Gyr. The other models yield age differences of $-5 \pm 6$ (Bruzual \& Charlot 2000), $-4 \pm 5$ Gyr (Kurth et al. 1999), and $-4 \pm 5$ Gyr (Vazdekis 1999). The mean age difference is $\langle\Delta t\rangle=-2 \pm 3$ Gyr. The two globular cluster sub-populations are coeval within $1 \sigma$.

With the assumption of coeval sub-populations we calculate the metallicity difference which is the driving parameter for the $V-K$ colour offset. Applying the model of Maraston (2001) yields $\Delta[\mathrm{Fe} / \mathrm{H}]=\left|[\mathrm{Fe} / \mathrm{H}]_{\text {blue }}-[\mathrm{Fe} / \mathrm{H}]_{\text {red }}\right|=1.48 \pm 0.57$ dex. The models of Bruzual \& Charlot, Kurth et al., and Vazdekis give $1.04 \pm 0.30,1.03 \pm 0.31$, and $0.99 \pm 0.19$, respectively. The weighted mean metallicity difference is $\Delta[\mathrm{Fe} / \mathrm{H}]=1.0 \pm$ 0.3 dex. The metallicity difference derived with the $[\mathrm{Fe} / \mathrm{H}]-$ $(V-K)$ relation from Paper I (see Sect. 3.1) is $1.28 \pm 0.32 \mathrm{dex}$ and is consistent within the errors with the model predictions.

\subsubsection{NGC 4365}

The NGC 4365 colour-colour diagram is more complex than the one for the NGC 3115 globular cluster system.

Dust: Can extinction by dust in NGC 4365 produce the colour sequence we observe? The reddening vector is plotted in the upper left corner of Fig. 6. If extinction by dust in NGC 4365 would play a role at all, one would expect this to have more impact within the galaxy body than in the halo, which would cause the bulge population to be systematically redder. Correcting for any additional internal reddening would shift the clusters along the reddening vector almost parallel to the iso-metallicity tracks towards younger ages (see Fig. 6). However, there is no evidence for the existence of significant amounts of dust in NGC 4365 (Jura et al. 1987; Goudfrooij et al. 1994).

Intermediate-age clusters: The most striking feature in Fig. 6 is a large population of globular clusters with colours that are red in $V-K(\sim 3.0)$ but intermediate in $V-I(\sim 1.0-1.2)$. According to SSP models this clusters fall in the regime of $\sim Z_{\odot}-3 Z_{\odot}$ metallicities and intermediate ages of $\sim 2-8 \mathrm{Gyr}$. In general, the red globular clusters appear on average younger than the blue sub-population ${ }^{7}$. With a few exceptions all

\footnotetext{
${ }^{7}$ We briefly review results of previous GCLF studies which were based on optical photometry only. From recent HST photometry of 500 globular clusters Larsen et al. (2001) find a difference in GCLF turn-over magnitudes $\Delta m_{\mathrm{TO}}=0.8 \pm 0.4$ mag between the red and blue sub-population which they cut at $V-I=1.05 \mathrm{mag}$. With the red clusters being fainter, their result implies that the red clusters are on
}

metal-rich $([\mathrm{Fe} / \mathrm{H}] \gtrsim-0.4$ dex $)$ clusters have $V-K, V-I$ colours indicative of intermediate to young ages. We estimate this intermediate-age clusters to represent $\sim 40-80 \%$ of our observed globular cluster sample. This limits are derived 1) by assuming all clusters red of the $[\mathrm{Fe} / \mathrm{H}]=-0.4$ dex iso-metallicity line (see Fig. 6) to be intermediate-age and 2) by taking into account the existing spread in colours of globular clusters in Local Group galaxies and only counting objects with colours redder than all Milky Way and M 31 globular clusters and with $V-K \gtrsim 3.0 \mathrm{mag}$ as intermediate-age clusters.

SSP models predict that the $V$ magnitude of a $\sim 2-8 \mathrm{Gyr}$ globular cluster is $\sim 1.7$ to 0.6 mag brighter than that of a 15 Gyr old cluster (e.g. Bruzual \& Charlot 2000). Thus we should be able to detect bright globular clusters in the CMD. The NGC 4365 CMD (lower panel in Fig. 3) shows indeed a population of relatively bright globular clusters around $21 \lesssim V \lesssim 22$ mag and $2.6 \lessgtr V-K \lesssim 3.4$ mag. Most of these objects, indeed, fall in the colour-colour diagram (see Fig. 6) into the regime of metal-rich intermediate-age populations with ages $\gtrsim 8 \mathrm{Gyr}$ and metallicities $Z \gtrsim Z_{\odot}$. As a consistency check we, conversely, select clusters in the colour-colour diagram (Fig. 6) with colours of metal-rich intermediate-age populations (see colour definitions above) and look for their $V$ magnitudes. They dominate the GCLF at brighter magnitudes: At $V \lesssim 22.0 \mathrm{mag} \sim 80 \%$ of all objects in the sample are metal-rich and have intermediate ages. Unfortunately, the small size and the colour bias of our sample does not allow to establish statistically whether or not the GCLF of the metalrich intermediate-age population is brighter than that of the remaining globular clusters.

Comparison of ages with known young clusters: Clusters of pre-AGB ages ( $\$ 100 \mathrm{Myr}$ ) would have not only different colours, but also considerably brighter magnitudes than the old population and would thus be easy to identify. A lower limit on the ages of the intermediate-age population can be set by comparing their colours with the data for very young $(\$ 1 \mathrm{Gyr})$ globular clusters in NGC 7252 (Maraston et al. 2001). All NGC 7252 clusters show too blue $V-I$ colours compared with the bulk of intermediate-age clusters in NGC 4365. This implies that the latter are at least $1 \mathrm{Gyr}$ old.

A recent study of globular clusters in NGC 1316 revealed an intermediate-age $(3 \pm 0.5 \mathrm{Gyr})$, solar-metallicity globular cluster sub-population (Goudfrooij et al. 2001a, b). The brightest and not-reddened intermediate-age cluster candidates have mean colours $V-I \approx 1.05$ and $V-K \approx 2.87$. This colours, compared with the mean locus of globular clusters in NGC 4365 (cf. Fig. 6), indicate that some of the intermediate-age globular clusters in NGC 4365 have counterparts in NGC 1316 which are $\sim 3$ Gyr old.

average older and more metal-rich than the blue sub-population, contrary to our findings. However, we note, that the $V-I$ colour distribution (see Fig. 4 in Larsen et al. 2001) is very broad with no apparent bimodality. Since Larsen et al.'s $V-I$ separation splits between blue and red clusters at roughly the colour of the intermediate-age globular cluster population, their GCLF analysis might be corrupted by the intermediate-age clusters that were not accounted for. 


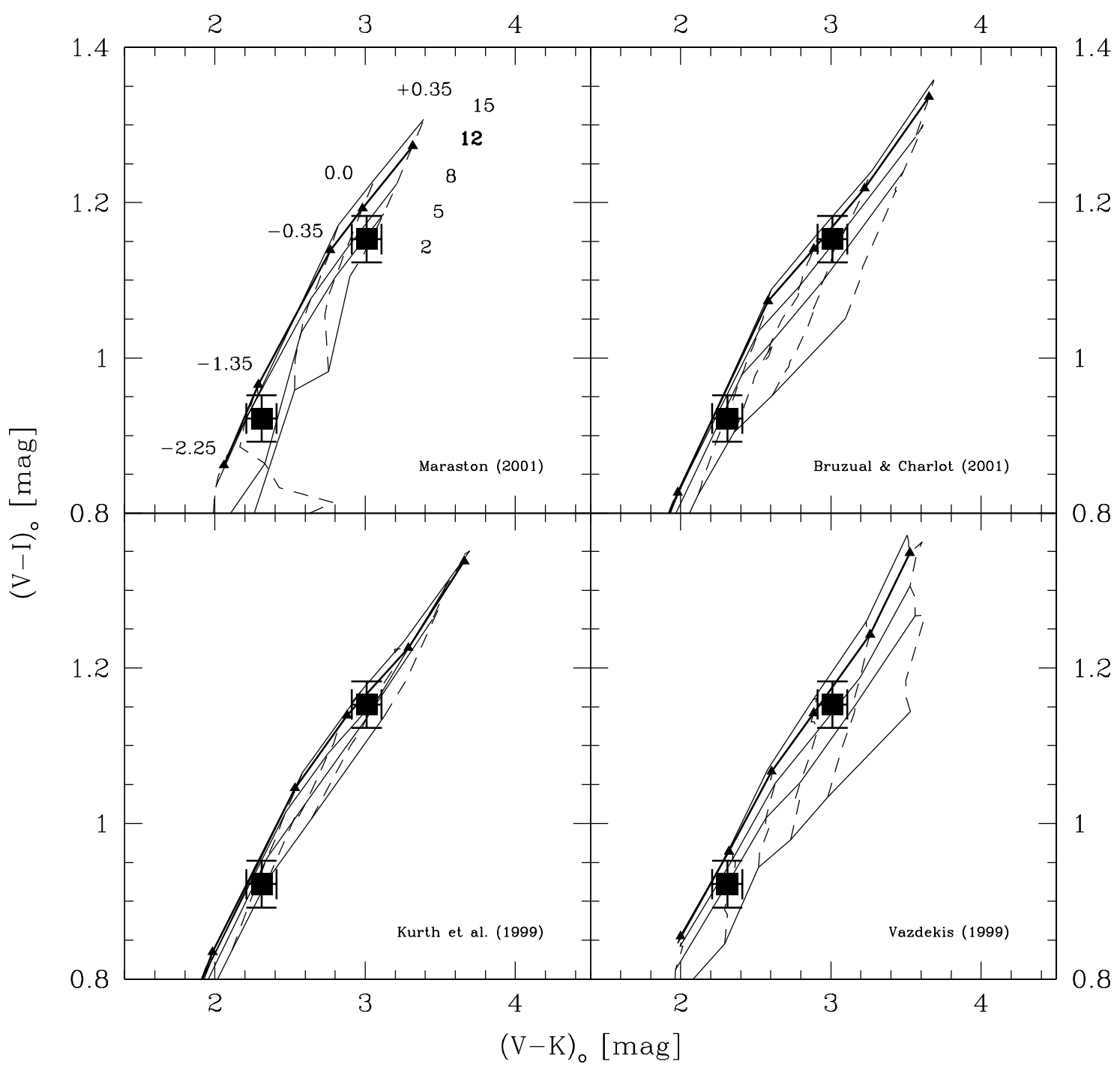

Fig. 7. NGC 3115: $V-I$ vs. $V-K$ colour-colour diagrams for major globular cluster sub-populations. The four panels show the mean colour of the two major globular cluster sub-populations in NGC 3115 with four different SSP model grid. In the upper left panel with the SSP model of Maraston (2001) we labeled iso-age (solid lines) and iso-metallicity tracks (dashed lines) in the range $2-15 \mathrm{Gyr}$ and $[\mathrm{Fe} / \mathrm{H}]=-2.25$ to +0.35 dex. All other model grids have exactly the same iso-age lines. For better comparison we plotted the 12 Gyr iso-age line thicker. Triangles show the position of the labeled metallicity along the 12 Gyr age track. The SSP models of Bruzual \& Charlot (2000); Kurth et al. (1999); Vazdekis (1999) have slightly different metallicity spacings. The are iso-metallicity tracks for $[\mathrm{Fe} / \mathrm{H}]=+0.4,0.0,-0.4,-0.7,-1.7$, and -2.3 dex starting at red colours (upper right in each panel).

Spatial distribution: Interestingly enough, the young globular clusters with colours $V-K \gtrsim 2.6$ seem to form a "bulge" or "disk" population, as the distribution of those clusters is clearly elongated along the major axis of the galaxy body (cf. Fig. 2). The major axis of the disk aligns with the major axis of the galaxy body. In contrast, blue globular clusters form a halo population around NGC 4365. Unfortunately, our field of view is concentrated to the south-east only and does not allow to check for consistency on the opposite side (north-west) of the major axis.

\subsection{Aging the NGC 4365 globular cluster system}

In the following we let the entire globular cluster system in NGC 4365 age for 10 Gyr and study their evolution in the colour-colour diagram. We assume a passive colour evolution due to age only. Each single globular cluster is aged individually for 10 Gyr.

Following Buzzoni (1995) a detailed inspection of each model shows that the parameter range $5 \lessgtr t \lesssim 15 \mathrm{Gyr}$ and $-0.7 \lesssim[\mathrm{Fe} / \mathrm{H}] \lesssim 0.4$ dex (except for Maraston models, for which we use a smaller metallicity range $-0.35 \lesssim[\mathrm{Fe} / \mathrm{H}] \lesssim$ 0.35 dex) allows a simple parameterization of colours by age and metallicity. The parameterization can be written as a first approximation:

$\Delta$ Colour $_{i}=\alpha_{i} \Delta \log t+\beta_{i} \Delta[\mathrm{Fe} / \mathrm{H}]$

where the slopes $\alpha_{i}, \beta_{i}$ are determined from the SSP models and are given in Table 4 . We use linear interpolation in each model grid and average the obtained ages and metallicities from all four models. For clusters with redder colours 


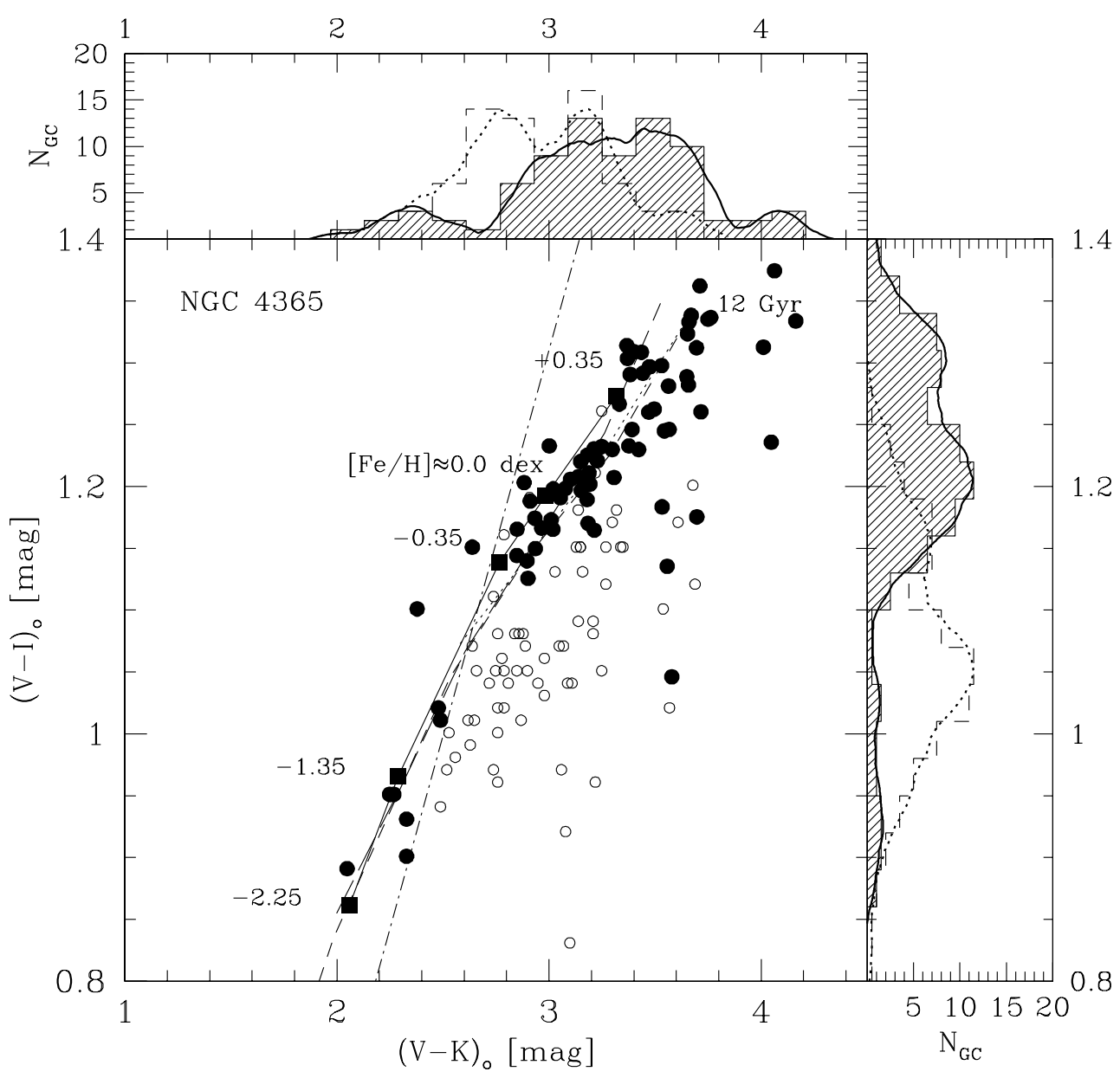

Fig. 8. NGC 4365: Colour-colour diagram for the evolved globular cluster system. Solid circles show the evolved data while open circles indicate the colours of the observed data set. Since an average evolution calculated from four SSP models was adopted we plot 12 Gyr iso-age tracks for each model with metallicities $-2.25<[\mathrm{Fe} / \mathrm{H}]<0.35$ dex. The metallicity sequence is indicated by solid squares. We used models from Maraston (2001) (solid), Kurth et al. (1999) (short-dashed), Bruzual \& Charlot (2000) (dotted), and Vazdekis (1999) (long-dashed). All clusters right of the dot-dashed line, which is the limit of the applied linear interpolation, were aged.

Table 4. Slopes $\alpha_{i}, \beta_{i}$ as determined from the SSP models. The mean $\alpha_{V-K}$ was calculated without the Vazdekis value.

\begin{tabular}{llcrr}
\hline \hline Model & $i$ & $\alpha_{i}=\partial / \partial(\log t)$ & $\beta_{i}=\partial / \partial[\mathrm{Fe} / \mathrm{H}]$ & parameter space \\
\hline Bruzual \& Charlot (2000) & $V-I$ & $0.239 \pm 0.029$ & $0.236 \pm 0.006$ & $5<t<15 \mathrm{Gyr}$ \\
& $V-K$ & $0.459 \pm 0.150$ & $0.964 \pm 0.015$ & $-0.7<[\mathrm{Fe} / \mathrm{H}]<+0.4$ \\
Kurth et al. (1999) & $V-I$ & $0.218 \pm 0.043$ & $0.259 \pm 0.011$ & $5<t<15 \mathrm{Gyr}$ \\
& $V-K$ & $0.529 \pm 0.092$ & $0.981 \pm 0.026$ & $-0.7<[\mathrm{Fe} / \mathrm{H}]<+0.4$ \\
Maraston (2001) & $V-I$ & $0.260 \pm 0.020$ & $0.207 \pm 0.011$ & $5<t<15 \mathrm{Gyr}$ \\
& $V-K$ & $0.554 \pm 0.017$ & $0.825 \pm 0.020$ & $-0.35<[\mathrm{Fe} / \mathrm{H}]<+0.35$ \\
Vazdekis (1999) & $V-I$ & $0.201 \pm 0.052$ & $0.265 \pm 0.006$ & $5<t<15 \mathrm{Gyr}$ \\
& $V-K$ & $0.065 \pm 0.133$ & $0.862 \pm 0.030$ & $-0.7<[\mathrm{Fe} / \mathrm{H}]<+0.4$ \\
\hline$\langle$ mean〉 & $V-I$ & $0.230 \pm 0.026$ & $0.242 \pm 0.026$ & $5<t<15 \mathrm{Gyr}$ \\
& $V-K$ & $0.514 \pm 0.040$ & $0.908 \pm 0.076$ & $-0.35<[\mathrm{Fe} / \mathrm{H}]<+0.35$ \\
\hline
\end{tabular}

than the model parameter space (see Table 4) we use an extrapolation to high-metallicities, being aware of the insecure prediction power of SSP models in this range. Clusters which have bluer colours than the model parameter space are dropped from the evolution due to apparent non-linearities in the grids beyond $[\mathrm{Fe} / \mathrm{H}] \lesssim-0.7$ dex and an expected small colour evolution. Since we assume passive evolution one can write

$\Delta(V-I)=0.230 \log \left(1+\frac{10 \mathrm{Gyr}}{t}\right)$ 
$\Delta(V-K)=0.514 \log \left(1+\frac{10 \mathrm{Gyr}}{t}\right)$

where $t$ is the current age of each single globular cluster determined from the colour-colour diagram and $\Delta(V-I)$ and $\Delta(V-K)$ are the colour changes over a $10 \mathrm{Gyr}$ lifetime. The mathematical form of these equations implies a rapid evolution in colour for intermediate-age globular cluster. As the clusters get older, the colour evolution slows down, i.e. old clusters are hardly affected in colour by the aging procedure.

We emphasize again that both upper equations are only valid for stellar populations older than $\sim 5$ Gyr (see Table 4) and that, especially for younger ages, the relations represent insecure extrapolations. Figure 8 shows the evolution of the colour-colour diagram and the according colour distributions.

Only clusters which fall right to the dot-dashed line are considered in the aging process. This boundary is defined by the limitation of our parameter space (see Table 4). Moreover, there are very few objects left of the line for which the models predict almost no colour evolution. The aging shows that the $10 \mathrm{Gyr}$ older globular cluster system (solid dots) contains a significant fraction of clusters with $Z>Z_{\odot}$ metallicities. Clusters with extremely red colours $V-K>4$, which correspond to metallicities $Z \gtrsim 10 Z_{\odot}$, are present in the new colour-colour distribution. Note, however that these extreme colours are the predictions of the extrapolated parameter range and might change when detailed models of extremely metal-rich stellar populations are available.

The aged $V-I$ and $V-K$ colour distributions (thick lines Fig. 8) show a very broad flat-top red peak. This broad peak suggests a wider metallicity dispersion than seen in the metalrich sub-system of Galactic globular clusters, which has a width of about 0.3 dex. This metallicity width translates into a scatter of $\sim 0.25 \mathrm{mag}$ in $V-K$ and $\sim 0.15 \mathrm{mag}$ in $V-I$. The red peak of the evolved intermediate-age clusters in NGC 4365 is at least twice as broad. However, both colour distributions, although statistically insignificant, show hints for a substructure of the red peak which might consist of two or more red sub-populations (see the $V-K$ colour histogram in Fig. 8).

The intermediate-age sub-population of today's NGC 4365 globular clusters will mature to colours which are comparable to colours of the red (metal-rich) sub-population in M 87 (Paper I). These broad red sub-populations are also found in other giant elliptical galaxies, such as NGC 4472 (e.g. Geisler et al. 1996; Puzia et al. 1999), NGC 3585, NGC 4526, NGC 4649, NGC 5846 (e.g. Gebhardt \& Kissler-Patig 1999; Larsen et al. 2001; Kundu \& Whitmore 2001a, b).

\subsection{Comparison with the integrated light of the host}

Figure 9 shows the $V-I$ and $V-K$ colours of the integrated light of NGC 3115 and NGC 4365 along with the colours of their globular cluster systems. The effective $V-I$ colour was taken from Buta \& Williams (1995). The $V-K$ colour was measured by Frogel et al. (1978) in different apertures which are not the effective apertures. We use the colour given by the aperture which is closest to the effective radius and calculate the expected change to an aperture of effective radius. All values are documented in Table 1.

From the comparison with SSP models both galaxies show diffuse stellar light colours which are consistent with a $\sim 12 \mathrm{Gyr}$ old $Z>Z_{\odot}$ metallicity stellar population. However, the observed colours are luminosity-weighted averages and can be reproduced by many different mixes of stellar populations with a wide range of ages and metallicities. The major difference between the two galaxies appears when the stellar light is compared with their globular cluster systems (see Fig. 9). The globular clusters in NGC 3115 seem to have the same age as the galaxy body. The colours of the integrated light fall onto the metallicity sequence which is set up by the globular clusters. A few very red globular clusters share the colours of the stellar light. This clusters appear to be associated with the stellar disk of NGC 3115.

In NGC 4365, the globular cluster system shows features that are not readily apparent in the diffuse stellar light. The galaxy's average stellar population is metal-rich and relatively old and the clusters span a wide range of metallicities and ages.

The triaxial galaxy NGC 4365 hosts a decoupled core which rotates around the minor-axis (Wagner et al. 1988; Bender \& Surma 1992; Surma \& Bender 1995) while the main body rolls around its major axis. The first detailed abundance study found the innermost core stellar population being metalrich $\left(\gtrsim 2.3 Z_{\odot}\right)$, relatively young $(\$ 7 \pm 1.5 \mathrm{Gyr})$ and excessively enriched in $\alpha$-elements (Surma \& Bender 1995). Davies et al. (2001) performed a subsequent integral-field spectroscopic study covering the galaxy out to $\sim 3 \mathrm{kpc}$ and found the same age ( $\sim 14$ Gyr) for the two distinct components, solar metallicity and a rather mild $\alpha$-element enhancement of the core. However, their Fig. 2 shows that there is an age gradient in the very center $\left(r \leq 1.6^{\prime \prime}\right)$ where the innermost population is $\sim 12$ Gyr old. The quite significant difference in age between the former two studies can be attributed to the use of two different SSP models.

The index measurements of both studies for $\mathrm{H} \beta$ and $\mathrm{MgI}$ are consistent with each other. In particular, while Surma \& Bender use Worthey (1992) models, Davies et al. (2001) use the models of (Vazdekis 1999) which, in contrast to Worthey's models, include late evolutionary phases, such as the HB (horizontal branch), AGB, EAGB, and PAGB. Since the HB can significantly contribute to the $\mathrm{H} \beta$ flux (Maraston \& Thomas 2000), which is commonly used as an age-sensitive index, the predictions of a model which includes the HB will yield higher ages for same $\mathrm{H} \beta$ measurements as models without $\mathrm{HB}$ modeling. The difference in age between the previous two studies can therefore be fully understood from the choice of models.

The latest Vazdekis99 models are also used in this study. Therefore, the spectroscopic and photometric predictions for age and metallicity can directly be compared with each other. For NGC 4365, with the photometric VIK values of Table 1 we obtain an age of $12 \pm 4$ Gyr and roughly solar metallicity. Considering that the photometric values are an average over a large radial range, the predictions of photometric and spectroscopic parameters from the Vazdekis99 models are consistent with each other. 


\subsection{Comparison with the Milky Way and M 31 globular clusters}

We compare the colour-colour diagrams of NGC 3115 and NGC 4365 clusters with the ones of Milky Way and M 31 globular clusters. Figure 9 shows the comparison of the colourcolour diagrams. The globular cluster system of NGC 3115 compares well with the globular cluster system of either Local Group spiral. The globular cluster systems of NGC 3115, Milky Way, and M 31 appear to be on average old and roughly coeval.

The few detected blue clusters in NGC 4365 show good agreement with the mean locus of metal-poor clusters in M 31 and the Milky Way. The NGC 4365 globular cluster system extends also to much higher $V-K$ at similar $V-I$ colours than the Local Group systems, implying high metallicities and intermediate to young ages (see also Fig. 6).

\section{Discussion}

\subsection{NGC 4365, a cooling-down galaxy}

Davies et al. (2001) find a luminosity-weighted age of $\sim 12 \mathrm{Gyr}$ for the solar-metallicity kinematically-decoupled core (KDC) in NGC 4365 using the diagnostic plot $(\mathrm{H} \beta$ vs. $[\mathrm{MgFe}])$ and SSP models of Vazdekis (1999). From the comparison of our near-IR/optical colours with the same models, we find that $\sim 40-80 \%$ of our sample are metal-rich $\left(Z \gtrsim 0.5 Z_{\odot}\right)$ globular clusters with ages between $\sim 2-8$ Gyr. However, within our observational measurement errors the age distribution, derived from the linear interpolation of SSP models, is consistent with a single-burst of globular-cluster formation which took place 3-4 Gyr ago. Together with the metal-rich globular clusters a part of the stellar population in the KDC must have been formed from the same pre-enriched gas in a significant star-formation event. In fact, Davies et al. (2001) find that the $\sim 2$ Gyr younger luminosity-weighted age of the KDC (compared to the outer parts of the galaxy) can be accounted for by a contaminating, 5 Gyr old stellar population of solar metallicity. This young population would amount $6 \%$ of the mass inside $r \lesssim 1.6^{\prime \prime}$ or $\lesssim 160$ pc.

It is tempting to associate the intermediate-age globular cluster population with the kinematically decoupled core. Within the prediction accuracy of SSP models this young KDC population is consistent with being the diffuse counterpart of the stellar population in metal-rich intermediate-age globular clusters. Numerical simulations of gaseous mergers (see e.g. Barnes \& Hernquist 1992, for a review) predict the settling of the progenitors' gas into the inner parts of the merger remnant within $\sim 10^{8}$ years. If globular clusters and the central stellar population were in fact formed from the same gas, both stellar populations have to be almost coeval.

We can compare the stellar mass contained in an intermediate-age component in NGC 4365 with the mass of the intermediate-age globular cluster population to test how consistent these are with forming in the same event. To calculate the mass of the diffuse stellar component, we begin with the decoupled core. Davies et al. found that the intermediate-age component makes up $6 \%$ of the stellar mass of the decoupled core. Given the total stellar mass estimate for the core of $M_{\text {core }} \lesssim(8.1 \pm 2.5) \times 10^{9} M_{\odot}$ from Surma \& Bender (1995), the resulting mass in the core of the intermediate-age diffuse stellar component is $(4.9 \pm 2.5) \times 10^{8} M_{\odot}$.

This mass is a lower limit to the total mass of the diffuse intermediate-age stellar population because it is unlikely that this component is found only in the very central region. In particular, the intermediate-age globular clusters are observed over a fairly extended region, and it would be difficult to arrange for the corresponding diffuse component not to have a similar extended spatial distribution. Therefore, in order to estimate the total stellar mass of the galaxy at intermediate ages, we need to estimate the fraction of the total galaxy mass coming from the intermediate age population. This fraction can not be greater than a few percent, or the extended intermediate-age population would have been detected by Davies et al. as they detected such a component in the central region. Conversely, the mass fraction of the intermediate-age component must be at least a few-tenths of a percent based on comparing the intermediateage stellar mass of $(4.9 \pm 2.5) \times 10^{8} M_{\odot}$ observed in the core to the total galaxy stellar mass of $\sim 4.8 \times 10^{11} M_{\odot}$ (Bender et al. 1989).

To bracket these possibilities, we carry out a representative calculation with a $1 \%$ fraction of the overall stellar mass of the galaxy in an intermediate-age component, and show how the results are affected by varying this in either direction. This fraction gives a stellar mass over the whole galaxy in the intermediate-age component of $\sim 5.2 \times 10^{9} \mathrm{M}_{\odot}$.

What is the corresponding mass currently in the intermediate-age GC system? After correcting our sample for incompleteness in luminosity and spatial coverage and with the assumption that $\sim 40-80 \%$ of our sample globular clusters have intermediate-ages, we find that the total number of intermediate-age globular clusters in NGC 4365 is $\sim 150$ (within a factor of 2). To determine the mass in this system, we adopt the two-component mass function of McLaughlin \& Pudritz (1996) over the mass range of $10^{4}-10^{8} M_{\odot}$, appropriate for the GCs of giant elliptical galaxies. This gives a total mass of $\sim 1.6 \times 10^{8} M_{\odot}$ in the globular cluster system. A similar result is found by simply counting up the estimated mass of the observed clusters and accounting for the spatial areas not covered by our observations.

The efficiency of formation and survival to several Gyr of the intermediate-age globular cluster system is $3 \%$. This follows directly from the derived mass of $\sim 1.6 \times 10^{8} M_{\odot}$ for the intermediate-age globular cluster system and the corresponding $\sim 5.2 \times 10^{9} M_{\odot}$ for the diffuse stellar component of the intermediate-age system. If the adopted mass fraction of the galaxy is too high, the formation and survival efficiency increases to rather high values of $10 \%$ or more. If the adopted mass fraction of the galaxy in intermediate-age component is a factor of a few too low, this number drops to about $1 \%$. It is difficult to make the data consistent with a formation efficiency less than $1 \%$.

How do these formation and survival efficiencies compare to other observations? Observations of strongly starbursting systems show that $\sim 20 \%$ of the stars form in compact star 

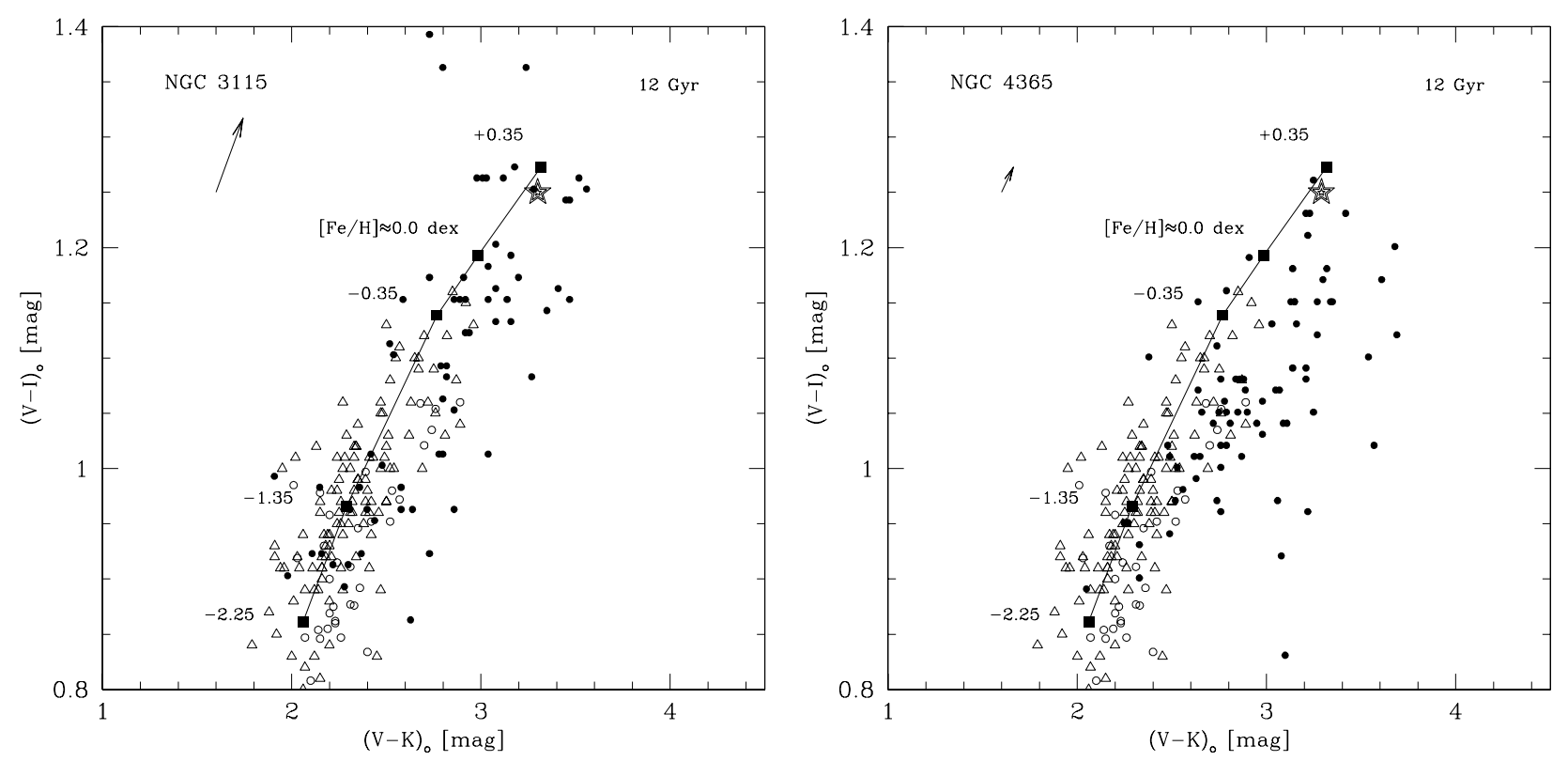

Fig. 9. Comparison of globular clusters in NGC 3115 (left panel) and NGC 4365 (right panel) with globular clusters in the Milky Way and M 31. Milky Way and M 31 clusters are shown by open circles and open triangles. The stars show the colour of the diffuse stellar light (see Table 1). As a guide to the eye, we plot a $12 \mathrm{Gyr}$ iso-age line for different metallicities in the range $-2.25 \leq[\mathrm{Fe} / \mathrm{H}] \leq+0.35 \mathrm{dex}$ taken from Maraston (2001). The metallicity is increasing to redder colours and is indicated by solid squares. Note, that in the two Local Group spirals the high-metallicity range contains only few globular clusters which are, moreover, prone to have inaccurate reddening corrections.

clusters (e.g. Meurer et al. 1995; Whitmore et al. 1999; Larsen \& Richtler 1999; Zepf et al. 1999). This represents the peak formation efficiency of globular clusters. Studies of older mergers find that a few percent of the stars are in globular clusters (e.g. Schweizer et al. 1996; Miller et al. 1997; Zepf et al. 1999; Goudfrooij et al. 2001b). The best determined case for older globular cluster systems is probably the Galactic halo, in which about $1 \%$ of the stars are in globular clusters. A global average of all spheroidal galaxy systems is around $0.3 \%$ (e.g. McLaughlin 1999). However, there are large variations between galaxies, and within sub-populations in individual galaxies. Dynamical evolution may significantly reduce the number of globular clusters observed at old ages compared to initial values at young ages (e.g. Gnedin \& Ostriker 1997; Vesperini 2000).

The comparison of the formation and survival efficiency of the NGC 4365 intermediate-age globular cluster system to other systems suggests that NGC 4365 and older mergers have about $2 \%$ of their stars in globular clusters, with an uncertainty of a factor of a few. It is possible that the stellar mass attributed to the younger component should be restricted to that in the core alone, in which case the formation and survival efficiency of the NGC 4365 globular cluster system would be more like the peak efficiency seen in recent mergers with little subsequent dynamical evolution. Underestimating the mass in the diffuse intermediate-age population would reduce the formation and survival efficiency by a factor of a few, but it is difficult to make the data consistent with globular cluster formation efficiencies less than a percent or so.

We can also compare the metallicity of the intermediateage globular cluster and diffuse stellar components. NGC 4365 shows super-metal-rich globular clusters $\left(Z>2 Z_{\odot}\right)$ while the luminosity-weighted stellar light of the decoupled core reveals a roughly solar metallicity (Surma \& Bender 1995; Davies et al. 2001). This is likely due to a mixing effect of the integrated light of stellar populations in the core. If stars and globular clusters were formed from the same pre-enriched material, super-solar metallicities are expected in the core, as well. The small fraction of light from super-metal-rich stars is likely to be washed out by insufficient resolution of the inner structures. With a spatial resolution of $\sim 1.4^{\prime \prime}$ (radial size of the decoupled core $\sim 8^{\prime \prime}$ ) Surma $\&$ Bender (1995) report that the metallicity is at least $2.3 Z_{\odot}$ in the center of NGC 4365. At face value, the core metallicity and the metallicity of the most metal-rich globular clusters are consistent.

\subsection{NGC 3115, a quiescent galaxy}

It seems that the colour difference between the two major subpopulations of globular clusters in NGC 3115 is mainly due to a difference in metallicity, rather than in age. Within the errors this result is still consistent with the findings of Kundu \& Whitmore (1998) who report, using optical photometry only, that the metal-rich clusters are about $\sim 3$ Gyr younger than the metal-poor ones.

In contrast to NGC 4365, NGC 3115 seems to have experienced no major star-formation event in the last $\sim 10 \mathrm{Gyr}$. Both its globular cluster sub-populations are roughly that old or older. This resembles the situation in the globular cluster system of the Milky Way, where the two sub-systems, corresponding to thick-disk/bulge and halo populations, differ mainly in metallicity (Minniti 1995; Côté et al. 2000, etc.) with a relatively small age difference where the metal-rich clusters may be 
slightly younger than their metal-poor counterparts (Rosenberg et al. 1999).

Quantitatively, in NGC 3115 it remains to be seen whether or not the specific frequency $S_{\mathrm{N}}$ (number of globular clusters per unit luminosity, see Harris \& van den Bergh 1981) for the metal-poor stellar populations (i.e., the ratio of metal-poor globular clusters to metal-poor halo stars) is also roughly three times higher than for the metal-rich stellar populations, as it is observed in NGC 5128 and M 31.

Given the limited information about the halo stars, we conclude that the globular cluster system and the majority of halo stars in NGC 3115 most likely formed in two epochs and/or by two different mechanisms more than $\sim 10 \mathrm{Gyr}$ ago. Ever since then, the stellar populations in globular clusters and in the galaxy halo seem to have undergone a purely passive evolution.

\section{Summary}

Using accurate optical and near infrared photometry of the globular cluster systems in NGC 3115 and NGC 4365 we found the following:

- The large color baseline introduced by the new $K$-band data allowed us to find, for the first time, a very metalrich intermediate-age population of globular clusters in NGC 4365. The clusters have ages between $\sim 2-8 \mathrm{Gyr}$ and metallicities $\sim 0.5 Z_{\odot}-3 Z_{\odot}$. They form a flattened population, which is aligned with the galaxy's major-axis, while the metal-poor clusters populate the halo. We emphasize that such intermediate-age populations can only be found from photometric data by obtaining deep $K$-band data in addition to high-quality optical imaging from HST/WFPC2.

- After 10 Gyr of passive evolution these globular clusters will show colours similar to super metal-rich globular clusters in giant elliptical cluster galaxies, such as M 87.

- NGC 3115 hosts an old and bimodal globular cluster population. From comparison to four different SSP models we found the blue and the red globular cluster sub-populations being coeval to within the errors $( \pm 3 \mathrm{Gyr})$.

- We determine a metallicity difference between the red (metal-rich) and blue (metal-poor) globular cluster subpopulation in NGC 3115 as $\Delta[\mathrm{Fe} / \mathrm{H}]=1.0 \pm 0.3 \mathrm{dex}$. Thus, for this particular globular cluster system, metallicity is primarily responsible for the bimodality.

Acknowledgements. We would like to thank the ESO user support group and the ESO science operations group for having carried out very successfully our programme in service mode. We thank Judy Cohen for providing near-IR data for Milky Way globular clusters. We are also grateful to Stephane Charlot and Claudia Maraston for providing their stellar population models prior to publication. THP gratefully acknowledges the financial support during his visits at Yale University where parts of this work were performed. THP also acknowledges the support by the German Deutsche Forschungsgemeinschaft, DFG project number Be 1091/10-1. SEZ acknowledges support for this work from NASA LTSA grant NAG5-11319. MH acknowledges support through Proyecto Fondecyt 3980032. DM is supported by FONDAP Center for Astrophysics and FONDECYT. MH,
DM, and PG thank the European Southern Observatory for supporting visits during which part of this work was carried out. PG was affiliated with the Astrophysics Division of the Space Science Department of the European Space Agency during part of this project.

\section{References}

Aaronson, M., \& Malkan, M., in preparation

Ashman, K. M., Bird, C. M., \& Zepf, S. E. 1994, AJ, 108, 2348

Ashman, K. M., \& Zepf, S. E. 1998, Globular Cluster Systems (Cambridge University Press)

Barmby, P., Huchra, J. P., Brodie, J. P., et al. 2000, AJ, 119, 727

Barnes, J. E., \& Hernquist, L. 1992, ARA\&A, 30, 705

Barth, A. J., Ho, L. C., Filippenko, A. V., \& Sargent, W. L. 1995, AJ, 110, 1009

Bender, R., Surma, P., Doebereiner, S., Moellenhoff, C., \& Madejsky, R. 1989, A\&A, 217, 35

Bender, R., \& Surma, P. 1992, A\&A, 258, 250

Bertin, E., \& Arnouts, S. 1996, A\&AS, 117, 393

Brandl, B., Sams, B. J., Bertoldi, F., et al. 1996, ApJ, 466, 254

Bruzual, A. G., \& Charlot, S. 2000, private communication

Buta, R., \& Williams, K. L. 1995, AJ, 109, 543

Buzzoni, A. 1995, ApJS, 98, 69

Cardelli, J. A., Clayton, G. C., \& Mathis, J. S. 1989, ApJ, 345, 245

Côté, P., Marzke, R. O., West, M. J., \& Minniti, D. 2000, ApJ, 533, 869

Daddi, E., Cimatti, A., Pozzetti, L., et al. 2000, A\&A, 361, 535

Davies, R. L., Kuntschner, H., Emsellem, E., et al. 2001, ApJ, 548, L33

Forbes, D. A. 1996, AJ, 112, 954

Frogel, J. A., Persson, S. E., Matthews, K., \& Aaronson, M. 1978, ApJ, 220, 75

Gebhardt, K., \& Kissler-Patig, M. 1999, AJ, 118, 1526

Geisler, D., Lee, M. G., \& Kim, E. 1996, AJ, 111, 1529

Gnedin, O. Y., \& Ostriker, J. P. 1997, ApJ, 474, 223

Goudfrooij, P., Hansen, L., Jorgensen, H. E., \& Norgaard-Nielsen, H. U. 1994, A\&AS, 105, 341

Goudfrooij, P., Mack, J., Kissler-Patig, M., Meylan, G., \& Minniti, D. 2001a, MNRAS, 322, 643

Goudfrooij, P., Alonso, M. V., Maraston, C., \& Minniti, D. 2001b, MNRAS, 328, 237

Harris, W. E. 1996, AJ, 112, 1487

Harris, W. E. 2001, Star Clusters, Saas-Fee Advanced Course (Springer), 28, 223

Harris, W. E., \& van den Bergh, S. 1981, AJ, 86, 1627

Holtzman, J. A., Faber, S. M., Shaya, E. J., et al. 1992, AJ, 103, 691

Holtzman, J., Burrows, C. J., Casertano, S., et al. 1995, PASP, 107, 1065

Hunter, D. A., O’Connell, R. W., Gallagher, J. S., \& Smecker-Hane, T. A. $2000, A J, 120,2383$

Jones, L. A., \& Worthey, G. 1995, ApJ, 446, L31

Jura, M., Kim, D. W., Knapp, G. R., \& Guhathakurta, P. 1987, ApJ, 312, L11

King, I. 1962, AJ, 67, 471

Kissler-Patig, M., Richtler, T., Storm, J., \& della Valle, M. 1997, A\&A, 327, 503

Kissler-Patig, M., Forbes, D. A., \& Minniti, D. 1998, MNRAS, 298, 1123

Kissler-Patig, M. 2000, Rev. Mod. Astron., 13, 13

Kissler-Patig, M., Brodie, J. P., \& Minitti, D. 2002, A\&A, 391, 441 (Paper I)

Kundu, A., \& Whitmore, B. C. 1998, AJ, 116, 2841

Kundu, A., Whitmore, B. C., Sparks, W. B., et al. 1999, ApJ, 513, 733 
Kundu, A., \& Whitmore, B. C. 2001a, AJ, 121, 2950

Kundu, A., \& Whitmore, B. C. 2001b, AJ, 122, 1251

Kurth, O. M., Fritze-v. Alvensleben, U., \& Fricke, K. J. 1999, A\&AS, 138,19

Larsen, S. S., \& Richtler, T. 1999, A\&A, 345, 59

Larsen, S. S., \& Brodie, J. P. 2000, AJ, 120, 2938

Larsen, S. S., Brodie, J. P., Huchra, J. P., Forbes, D. A., \& Grillmair, C. J. 2001, AJ, 121, 2974

Liu, M. C., Dey, A., Graham, J. R., et al. 2000, AJ, 119, 2556

Maraston, C., \& Thomas, D. 2000, ApJ, 541, 126

Maraston, C., Kissler-Patig, M., Brodie, J. P., Barmby, P., \& Huchra, J. P. 2001, A\&A, 370, 176

Maraston, C. 2001, private communication

McLaughlin, D. E. 1999, AJ, 117, 2398

McLaughlin, D. E., \& Pudritz, R. E. 1996, ApJ, 457, 578

Mei, S., Kissler-Patig, M., Silva, D. R., \& Quinn, P. J. 2001, A\&A, 376, 793

Meurer, G. R., Heckman, T. M., Leitherer, C., et al. 1995, AJ, 110, 2665

Miller, B. W., Whitmore, B. C., Schweizer, F., \& Fall, S. M. 1997, AJ, 114,2381

Minniti, D. 1995, AJ, 109, 1663

Moorwood, A., Cuby, J.-G., Biereichel, P., et al. 1998, The Messenger, 94,7

O'Connell, R. W., Gallagher, J. S., \& Hunter, D. A. 1994, ApJ, 433, 65

O'Connell, R. W., Gallagher, J. S., Hunter, D. A., \& Colley, W. N. 1995, ApJ, 446, L1

Olszewski, E. W., Suntzeff, N. B., \& Mateo, M. 1996, ARA\&A, 34, 511

Persson, S. E., Murphy, D. C., Krzeminski, W., Roth, M., \& Rieke, M. J. 1998, AJ, 116, 2475

Puzia, T. H., Kissler-Patig, M., Brodie, J. P., \& Huchra, J. P. 1999, AJ, 118,2734

Puzia, T. H., Kissler-Patig, M., Brodie, J. P., \& Schroder, L. L. 2000a, AJ, 120, 777

Puzia, T. H., Kissler-Patig, M., Brodie, J. P., \& Huchra, J. P. 2000b, AJ, 120, 1160
Puzia, T. H. et al. 2002, IAU Symp., 207, in press

Renzini, A. 1981, Ann. Phys. Fr., 6, 87

Robin, A. C., Haywood, M., Crézé, M., Ojha, D. K., \& Bienaymé, O. 1996, A\&A, 305, 125

Rosenberg, A., Saviane, I., Piotto, G., \& Aparicio, A. 1999, AJ, 118, 2306

Saviane, I., Rosenberg, A., Piotto, G., \& Aparicio, A. 2000, A\&A, 355,966

Schlegel, D. J., Finkbeiner, D. P., \& Davis, M. 1998, ApJ, 500, 525

Schweizer, F., Miller, B. W., Whitmore, B. C., \& Fall, S. M. 1996, AJ, 112,1839

Silverman, B. W. 1986, Density Estimation for Statistics and Data Analysis (Chap and Hall/CRC Press, Inc.)

Surma, P., \& Bender, R. 1995, A\&A, 298, 405

Tonry, J., \& Schneider, D. P. 1988, AJ, 96, 807

Tonry, J. L., Dressler, A., Blakeslee, J. P., et al. 2001, ApJ, 546, 681 van den Bergh, S. 2000, PASP, 112, 932

de Vaucouleurs, G., de Vaucouleurs, A., Corwin, J. R., et al. 1991, Third reference catalogue of Bright galaxies (New York: SpringerVerlag)

Vazdekis, A. 1999, ApJ, 513, 224

Vazdekis, A., \& Arimoto, N. 1999, ApJ, 525, 144

Vesperini, E. 2000, MNRAS, 318, 841

Wagner, S. J., Bender, R., \& Moellenhoff, C. 1988, A\&A, 195, L5

Whitmore, B. C., Schweizer, F., Leitherer, C., Borne, K., \& Robert, C. 1993, AJ, 106, 1354

Whitmore, B. C., \& Schweizer, F. 1995, AJ, 109, 960

Whitmore, B. C., Miller, B. W., Schweizer, F., \& Fall, S. M. 1997, AJ, 114,1797

Whitmore, B. C., Zhang, Q., Leitherer, C., et al. 1999, AJ, 118, 1551

Worthey, G. 1992, Ph.D. Thesis

Worthey, G., \& Ottaviani, D. L. 1997, ApJS, 111, 377

Yi, S., Demarque, P., Kim, Y., et al. 2001, ApJS, 136, 417

Zepf, S. E., Ashman, K. M., English, J., Freeman, K. C., \& Sharples, R. M. 1999, AJ, 118, 752 